\title{
CLEAVER: CLASSIFICATION OF EVERYDAY ACTIVITIES VIA ENSEMBLE RECOGNIZERS
}

\author{
A Thesis \\ presented to \\ the Faculty of California Polytechnic State University, \\ San Luis Obispo
}

\author{
In Partial Fulfillment \\ of the Requirements for the Degree \\ Master of Science in Computer Science
}

by

Samantha Hsu

December 2018 
(c) 2018

Samantha Hsu

ALL RIGHTS RESERVED 


\section{COMMITTEE MEMBERSHIP}

TITLE:

CLEAVER: CLassification of Everyday Activities Via Ensemble Recognizers

AUTHOR:

Samantha Hsu

DATE SUBMITTED: December 2018

COMMITTEE CHAIR: Alexander Dekhtyar, Ph.D.

Professor of Computer Science

COMMiTteE MEMBER: Sarah Kozey Keadle, Ph.D., M.P.H.

Assistant Professor of Kinesiology and Public Health

COMMITTEE MEMBER: Franz J. Kurfess, Ph.D.

Professor of Computer Science 


\begin{abstract}
CLEAVER: CLassification of Everyday Activities Via Ensemble Recognizers
\end{abstract}

\author{
Samantha Hsu
}

Physical activity can have immediate and long-term benefits on health and reduce the risk for chronic diseases. Valid measures of physical activity are needed in order to improve our understanding of the exact relationship between physical activity and health. Activity monitors have become a standard for measuring physical activity; accelerometers in particular are widely used in research and consumer products because they are objective, inexpensive, and practical. Previous studies have experimented with different monitor placements and classification methods. However, the majority of these methods were developed using data collected in controlled, laboratory-based settings, which is not reliably representative of real life data. Therefore, more work is required to validate these methods in free-living settings.

For our work, 25 participants were directly observed by trained observers for two two-hour activity sessions over a seven day timespan. During the sessions, the participants wore accelerometers on the wrist, thigh, and chest. In this thesis, we tested a battery of machine learning techniques, including a hierarchical classification schema and a confusion matrix boosting method to predict activity type, activity intensity, and sedentary time in one-second intervals. To do this, we created a dataset containing almost 100 hours worth of observations from three sets of accelerometer data from an ActiGraph wrist monitor, a BioStampRC thigh monitor, and a BioStampRC chest monitor. Random forest and $k$-nearest neighbors are shown to consistently perform the best out of our traditional machine learning techniques. In addition, we reduce the severity of error from our traditional random forest classifiers on some monitors using a hierarchical classification approach, and combat the imbalanced nature of our dataset using a multi-class (confusion matrix) boosting method. Out of the 
three monitors, our models most accurately predict activity using either or both of the BioStamp accelerometers (with the exception of the chest BioStamp predicting sedentary time). Our results show that we outperform previous methods while still predicting behavior at a more granular level. 


\section{ACKNOWLEDGMENTS}

Thanks to:

- My family, for supporting me throughout my college career; none of my accomplishments would be possible without you. I love you.

- My advisor, Alex Dekhtyar, for consistently providing me feedback and direction. My thesis wouldn't have been as successful as it is without you.

- My second advisor, Dr. Sarah Keadle, for your advice, guidance, and DATA! This work would not have been possible without you.

- Jake Davis, for being my best friend and my number one supporter. You're amazing and I love you.

- Leanne and Ron Davis, for opening up their home to me and allowing me to stay with them while I finished my thesis. I will never know how to thank you enough.

- Nick Russo, for surviving this $4+1$ process with me. Slaving over our theses together has made this all the more bearable. You are great.

- My final committee member Dr. Franz Kurfess - I understand the time commitment that comes with being on a committee and am so grateful.

- All of my friends for their love and support and for cheering me on! 


\section{TABLE OF CONTENTS}

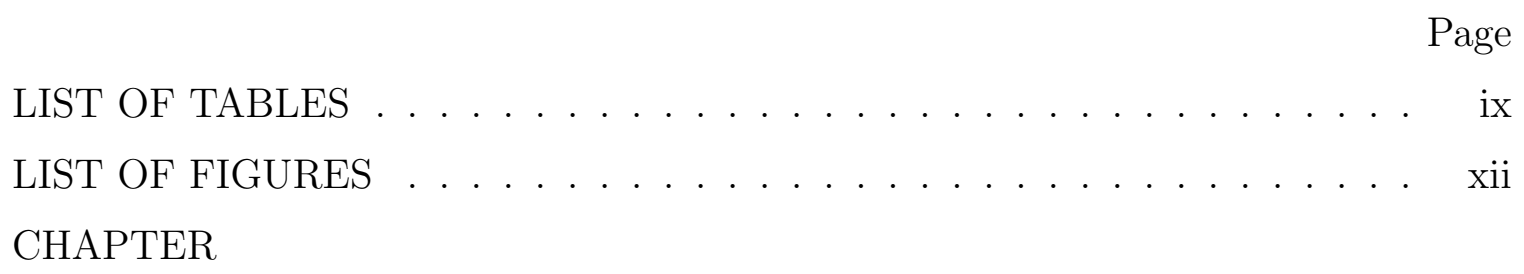

1 Introduction . . . . . . . . . . . . . . . . . 1

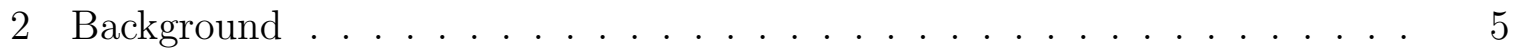

2.1 Classification . . . . . . . . . . . . . . . . 7

2.1.1 K-Nearest Neighbors . . . . . . . . . . . . . . 7

2.1.2 Support Vector Machine . . . . . . . . . . . . . . 9

2.1.3 Random Forests . . . . . . . . . . . . . . . . . . . . . . . 10

2.1.4 Boosting ........................ 10

2.1.5 Hierarchical Classification . . . . . . . . . . . . . 11

3 Related Work . . . . . . . . . . . . . . . . . . . 15

4 Design and Implementation . . . . . . . . . . . . . . . . . . . 18

4.1 Kinesiology Experimental Design . . . . . . . . . . . . 18

4.2 Dataset Construction . . . . . . . . . . . . . . . 20

4.2.1 Ground Truth. . . . . . . . . . . . . . 21

4.2.2 Raw Accelerometer Data. . . . . . . . . . . . . . . . . . 24

4.2.3 Merging Ground Truth with Features. . . . . . . . . . . . 27

4.2 .4 Final Dataset. . . . . . . . . . . . . . . . . . 28

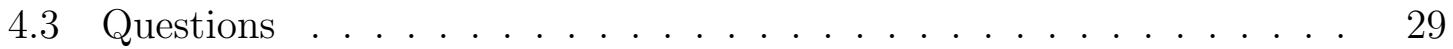

4.4 Experiments ......................... 30

4.4.1 K-Nearest Neighbors. . . . . . . . . . . . . . . . . 31

4.4.2 Support Vector Machine. . . . . . . . . . . . . . . 31

4.4 .3 Random Forest. . . . . . . . . . . . . . . . . . . . . . . . . . . 32

4.4.4 AdaBoost. . . . . . . . . . . . . . . . . 32

4.4.5 Gradient Boosting. . . . . . . . . . . . . . . . . 32

4.4.6 Hierarchical Classifier. . . . . . . . . . . . . . . . . . . 33

4.4.7 Confusion Matrix Boosting (CoMBo). . . . . . . . . . . . 34 
5 Results. . . . . . . . . . . . . . . . . . . . . . 37

5.1 Evaluation Methods . . . . . . . . . . . . . . . . 37

5.1 .1 Micro-level Evaluation . . . . . . . . . . . . . . . . 37

5.1 .2 Macro-level Evaluation . . . . . . . . . . . . . . . . . 37

$5.2 \quad$ Model Results . . . . . . . . . . . . . . . . . . . . . 38

5.2.1 Sedentary vs. Non-Sedentary Coding Results . . . . . . . . . 38

5.2 .2 Activity Intensity (METs) Results . . . . . . . . . . . . 41

5.2 .3 General Postures Results . . . . . . . . . . . . . . . . . . 44

5.2.4 Full Coding Scheme Results . . . . . . . . . . . . . . . . 46

6 Discussion and Conclusion . . . . . . . . . . . . . . . . 57

7 Future Work . . . . . . . . . . . . . . . . . . . . 61

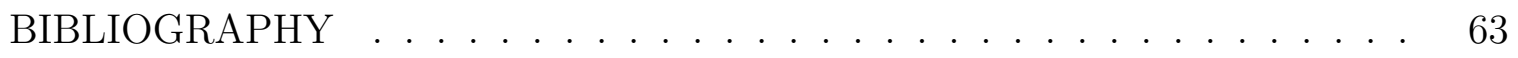
APPENDICES

A Dataset Samples . . . . . . . . . . . . . . . . 71

B Confusion Matrices . . . . . . . . . . . . . . . . . . 74

C Model Accuracies . . . . . . . . . . . . . . . . . . . . . 79 


\section{LIST OF TABLES}

Table

4.1 Activity domains and participant directions . . . . . . . . 19

4.2 Behavior coding options. . . . . . . . . . . . . . . . 20

4.3 Posture coding options with their upper body and intensity modifier options. . . . . . . . . . . . . . . . . 21

4.4 Columns in the provided ground truth criterion log. . . . . . . . . . 22

4.5 Columns in the second-by-second ground truth files. . . . . . . . . . 23

4.6 Additional statistical features used to summarize the accelerometer signals. . . . . . . . . . . . . . . . . 26

4.7 The new posture coding scheme. . . . . . . . . . . . . . . . 28

4.8 Different coding schemes. . . . . . . . . . . . . . . . . 31

5.1 Model performance predicting sedentary time using the 7 features from [56]. . . . . . . . . . . . . . . . . . . . . . . . . . . . . . . . . 38

5.2 Overall confusion matrix for RF predicting sedentary time from wrist Actigraph. . . . . . . . . . . . . . . .

5.3 Overall confusion matrix for RF predicting sedentary time from thigh and chest BioStamps. . . . . . . . . . . . . . . . . 39

5.4 Postures that fall under each intensity label. . . . . . . . . . . . 41

5.5 Model performance predicting activity intensity (METs) from aggregating our 14-class models. . . . . . . . . . . . . . . . 42

5.6 Classification accuracies predicting activity intensities (METs) from aggregating our 14-class models vs. our retrained models. . . . . . . 42

5.7 KL divergence of our aggregated 14-class models vs. our retrained models. . . . . . . . . . . . . . . . . . . . 44

5.8 Postures/behaviors that are considered for each general posture. . . 44

5.9 Model performance predicting the general coding scheme with 5 posture labels. . . . . . . . . . . . . . . . . . . . . .

5.10 Confusion matrix for RF predicting 5 general activity classes using the thigh and chest BioStamps. . . . . . . . . . . . . . . 46

5.11 Confusion matrix for $K \mathrm{NN}$ classifier predicting 5 general activity classes using wrist Actigraph. . . . . . . . . . . . 46

5.12 Model performance on the full coding scheme. . . . . . . . . . . . . 47 
5.13 Confusion matrix for our random forest model predicting the full coding scheme using the combined thigh and chest monitor data . .

5.14 Precision, recall, and f-measures of our thigh and chest BioStamps random forest, sorted by class proportion to the dataset. . . . . . .

5.15 Flat random forest classifier versus random forest ensemble hierarchy performance. . . . . . . . . . . . . . .

5.16 Confusion matrix for the random forest classifier predicting the first class level of the hierarchy from the combination of the thigh and chest monitor data. . . . . . . . . . . . . . . . . . . . . .

5.17 Confusion matrix for our hierarchical random forest ensemble predicting the full coding scheme using the combined thigh and chest monitor data . . . . . . . . . . . . . . . . 53

5.18 The ordinal coding of the posture classes. . . . . . . . . . 54

5.19 MA-MAE comparison of models. . . . . . . . . . . . 55

5.20 Preliminary CoMBo accuracies by activity domain for combined thigh and chest BioStamps. . . . . . . . . . . . . . 56

B.1 Confusion matrix for retrained random forest model predicting METs from wrist Actigraph. . . . . . . . . . . . . . . . . 74

B.2 Confusion matrix for retrained random forest model predicting METs from thigh BioStamp. . . . . . . . . . . . . . . . . . . . 74

B.3 Confusion matrix for retrained random forest predicting METs from chest BioStamp. . . . . . . . . . . . . . . . . 74

B.4 Confusion matrix for retrained random forest predicting METs from thigh and chest BioStamps. . . . . . . . . . . . . 75

B.5 Confusion matrix for RF predicting sedentary time from wrist Actigraph on active observation sessions. . . . . . . . . . . . . 75

B.6 Confusion matrix for RF predicting sedentary time from wrist Actigraph on errands observation sessions. . . . . . . . . . . 75

B.7 Confusion matrix for RF predicting sedentary time from wrist Actigraph on work observation sessions. . . . . . . . . . . 75

B.8 Confusion matrix for RF predicting sedentary time from wrist Actigraph on leisure observation sessions. . . . . . . . . . . . . 75

B.9 Confusion matrix for RF predicting sedentary time from wrist Actigraph on household observation sessions. . . . . . . . . . . . 76

B.10 Confusion matrix for RF predicting sedentary time from thigh and chest BioStamps on active observation sessions. . . . . . . . . . 76 
B.11 Confusion matrix for RF predicting sedentary time from thigh and chest BioStamps on errands observation sessions. . . . . . . . . 76

B.12 Confusion matrix for RF predicting sedentary time from thigh and chest BioStamps on work observation sessions. . . . . . . . . . 76

B.13 Confusion matrix for RF predicting sedentary time from thigh and chest BioStamps on leisure observation sessions. . . . . . . . . 76

B.14 Confusion matrix for RF predicting sedentary time from thigh and chest BioStamps on household observation sessions. . . . . . . . 77

B.15 Confusion matrix for AdaBoost predicting the full coding scheme on combined BioStamp thigh and chest monitor data. . . . . . . . 78

C.1 Test accuracy comparison of random forests with transition seconds in the dataset vs. excluding transition seconds from the dataset on the full coding scheme across all monitors. . . . . . . . . . . . . 79

C.2 Test accuracy comparison of random forests using different maximum tree depths on the sedentary coding scheme. . . . . . . . . . . 79

C.3 Test accuracy comparison of models with different number of total features on the sedentary coding scheme. . . . . . . . . . . . . 79 


\section{LIST OF FIGURES}

Figure

2.1 KNN example: The star will be classified by the majority vote of its $k$-nearest neighbors $[47] \ldots \ldots . \ldots . \ldots . \ldots$

2.2 Support Vector Machine hyperplane example [44] . . . . . . . . . . 9

2.3 Four types of hierarchical classification approaches [46] . . . . . . 12

2.4 Example of a hierarchical classification problem . . . . . . . . . 13

4.1 An example of the raw data from the Actigraph wrist monitor. . . 25

4.2 An example of the raw data from the BioStamp chest monitor. . . . 27

4.3 Our 3-tier class hierarchy. . . . . . . . . . . . . . . . . . 34

5.1 Actual by predicted sedentary time by activity domain. . . . . . . . 40

5.2 Actual vs. predicted probability distributions of random forests predicting activity intensity. . . . . . . . . . . . . . . . 43

5.3 Posture proportions to our dataset by their F-measures. . . . . . . 49

$5.4 \quad$ KL divergence of our random forest models. . . . . . . . . . . . . . 50

A.1 A snapshot of the direct observation timestamp log as provided by the Cal Poly Kinesiology and Public Health Department. . . . . . . 71

A.2 A snapshot of the ground truth criterion log as provided by the Cal Poly Kinesiology and Public Health Department. . . . . . . . . . . 72

A.3 Posture coding options and their respective modifier options. . . . . 73 


\section{Chapter 1}

\section{INTRODUCTION}

Physical activity is important for improving health and reducing risk for diseases such as cardiovascular disease, stroke, obesity, diabetes, metabolic syndrome, and some cancers $[5,55,28]$. Accurate measures of physical activity are vital to obtaining a better understanding of the dose-response relationship between physical activity and health [50]. Over recent years, considerable interest has grown in regards to the assessment of physical activity, and human activity monitoring and recognition has been widely studied, using wearable sensors called activity monitors that enable continuous monitoring $[55,36]$. Activity monitors are capable of evaluating activity type, duration, and intensity, and specifically accelerometer-based activity monitors have become the ideal measurement tool of choice. They are inexpensive, lightweight, and small enough so that they are unobtrusive to the participants wearing them for long periods of time, making objective activity monitoring practical. Accelerometers are widely used by researchers for assessing physical activity, especially for assessing free-living subjects (i.e., in real-world conditions). [15, 38, 57, 6, 11].

Objective physical activity assessment in a free-living environment is a necessity for a comprehensive understanding of the association between physical activity and health. There have been many successful physical activity classification studies with accelerometers in laboratory-controlled settings which enable the data to be of high quality $[19,21,38,6]$. However, there is evidence that the laboratory data does not accurately represent human behavior in a free-living, uncontrolled setting [27, 17]. Data collection in a controlled lab setting is also limited to short durations, which is unrealistic for real life applications when an individual would wear the activity monitor for longer periods of time [38]. 
Earlier work has implemented regression methods to model the relationship between accelerometer data and energy expenditure $[8,13,24]$. More recently, machine learning algorithms have been used in activity classification research, as they can fit a greater variety of activity metrics and provide better prediction accuracy than the regression techniques $[6,15,57]$. However, further exploration of machine learning methods needs to be done in this domain.

Dr. Sarah Kozey Keadle of the Cal Poly Kinesiology and Public Health Department has conducted research validating commercially available monitors for assessing sedentary behavior [33], validating two novel machine learning methods and a laboratory-calibrated neural network in a free-living environment [37], comparing hip and wrist accelerometer estimates of moderate-vigorous physical activity [59], and predicting sedentary behvavior from a wrist-worn accelerometer using machine learning [41]. However, these studies had small samples and did not ensure a range of activity types were included in the validation. To address this gap, Dr. Keadle recruited 25 subjects, who participated in two two-hour free-living activity sessions over a period of seven consecutive days. Participants wore accelerometers on the wrist, thigh, and chest, and were directly observed by the trained research assistants during these sessions. The direct observation served as the ground truth for this work; we combined the ground truth observation data with the raw accelerometer data from the three activity monitors to create our dataset.

The objective of this work is to predict an individual's physical activity/posture based on wrist, thigh, and chest accelerometer data. Dr. Keadle is particularly interested in investigating the following:

1. What monitor placement and machine learning method best determines sedentary vs. non-sedentary behavior?

2. How do our sedentary vs. non-sedentary models compare to previous methods? 
3. What monitor placement and machine learning method best estimates activity intensity level?

4. What monitor placement and classifier best predicts posture into 5 general posture classes?

5. What monitor placement and machine learning model best predicts posture/intensity into 14 posture classes?

While we were addressing Dr. Keadle's questions, we came up with a couple additional questions of our own. Specifically, we were interested in investigating new approaches to predict our most granular set of 14 class variables. Our additional questions are:

6. Does a hierarchical random forest ensemble improve classification accuracy for predicting 14 posture classes?

7. Does using a confusion matrix boosting method improve classification accuracy for predicting 14 posture classes?

To address our seven research questions, we used the activity monitor data and the ground truth observation data from the Cal Poly Kinesiology and Public Health Department and created a dataset on which we ran a battery of machine learning methods.

The contributions of this work are as follows:

- Development of the proper ground truth dataset.

- Testing a range of machine learning techniques on three novel sets of monitor data. 
- Demonstrating that the predictions of our most granular, 14-class models can be aggregated into 4 class variables and produce similar distributions to new models that have been retrained to learn the less granular coding scheme.

- A hierarchical classification schema that performs competitively with traditional classification models.

- Implementation of a multi-class boosting method that uses the confusion matrix as an error measure to better train classifiers on our imbalanced dataset.

- A collection of studies that address our seven research questions.

This document is organized as follows. Chapter 2 covers background information relevant to physical activity classification and machine learning methods. Then Chapter 3 explores related work in the field of physical activity recognition. Chapter 4 describes our experimental design and implementation. Results are presented in Chapter 5, and Chapter 6 discusses the conclusions of this study. Finally, Chapter 7 concludes with potential directions for future work. 


\section{Chapter 2}

\section{BACKGROUND}

Physical activity is one of the most beneficial things a person can do for their health [32]. Not only does it improve overall physical and mental health, but also reduces the risk of chronic noncommunicable diseases such as cardiovascular disease, obesity, diabetes, metabolic syndrome, and some types of cancer. Cardiovascular disease (CVD) is the leading cause of death in the world, killing 17.3 million per year - this figure is expected to rise to over 23.6 million by 2030 [2]. Obesity and diabetes are key risk factors associated with CVD and are also among the top risks and causes of global deaths. In the United States, $39.8 \%$ of adults are considered obese and $9.4 \%$ of the entire American population is diabetic $[10,1]$. As technology continues to influence modern lifestyle to become more sedentary and relatively inactive compared to previous generations, promoting an active lifestyle is crucial to improving health and reducing preventable deaths in the future. According to the American Heart Association's 2015 Heart Disease and Stroke Statistics Update, 31\% of U.S. adults report participating in no leisure time physical activity [2]. Objective and accurate methods of measuring physical activity are required in order to improve our understanding of the exact association between physical activity and specific health outcomes.

Traditionally, physical activity has been measured by self-report questionnaires. Although self-reports are easily administered, low-cost methods that can collect detailed information about an individual's physical activity, people tend to overestimate the amount of time they spend participating in vigorous activity, and underestimate the amount of time they spend participating in unstructured daily physical activity

(e.g., walking) [15]. Wearable activity monitors have been developed to objectively capture physical activity with respect to type, duration, and intensity by analyzing 
and quantifying human body movements. These activity monitors have advanced from only being able to evaluate the quantity of physical activity (e.g., pedometers), to activity recognition systems that are capable of assessing the quantity and quality of physical activity (e.g., fitness and activity trackers - Fitbit, Apple Watch, Garmin) [27]. Wearable activity sensors provide feedback about the user's routine with respect to physical activity and thus motivate a more active lifestyle [21]. Wearable accelerometers have been deemed the ideal choice for collecting measurements of physical activity and sedentary behavior. Their small dimensions and light weight allow them to be conveniently worn for extended periods of time while collecting data across multiple aspects of physical activity (i.e., total activity, time in different activity intensity levels, predicted energy expenditure) and remaining relatively inexpensive, making them the most widely studied in the activity recognition field.

Over recent decades, researchers have used classification algorithms with accelerometer data to measure and predict energy expenditure [57, 19], sedentary time[37], activity type and intensity [6], locomotion time [56], and other aspects of human activity. Earlier research focused on classifying activity from data collected in laboratory settings. Although the most common daily activities - sitting, standing, walking, and lying - have been successfully recognized with accelerometers [39, 22, 43, 23, 29, 40], it's been shown that experiments on laboratory data are not accurate indicators for how the classifiers perform on real-life data [27]. This is due to the fact that laboratory-collected data can potentially fail to represent behavior that happens outside of the laboratory. Studies using laboratory data tend to use data that cover minimal periods of time per activity - sitting, walking on a treadmill, or lying down for a number of seconds, for example. The amount of variability of movement during these couple of minutes in each activity is more likely to be reduced since the activity is only performed for a short period of time. This makes small postural changes, such as typing while sitting, less likely to be recorded because the sitting activity is only 
recorded for a few seconds. Because capturing real daily life data is essential to better understand and quantify the relationship between physical activity and specific health outcomes, it is important to evaluate free-living data to achieve valid classification accuracy. Researchers have experimented with a range of data processing methods for activity recognition. Earlier work has used simple regression methods to estimate energy expenditure [8, 13] and classify physical activity [24]. More recently, machine learning approaches have been explored in the physical activity recognition domain, and shown to outperform traditional regression methods $[15,6,57]$. Machine learning methods have the ability to capture more sophisticated dependencies and nonlinearities than simple regression methods; therefore, they can classify specific behaviors that cannot be characterized by simple linear relationships with acceleration data [19]. A variety of machine learning algorithms have been applied to physical activity classification, including support vector machines (SVMs) [53, 27, 38], random forests $[18,19]$, decision trees [6, 27], and artificial neural networks [15, 57].

\subsection{Classification}

The main objective of this work is to determine an individual's activity based on their movements collected from an ActiGraph wrist monitor, a BioStampRC thigh monitor, and a BioStampRC chest monitor. In attempt to do so, this work uses the following models: $k$-nearest neighbors, support vector machines (SVM), random forests, boosting algorithms, and a hierarchical ensemble.

\subsection{1 $\quad K$-Nearest Neighbors}

The $K$-Nearest Neighbors $(K \mathrm{NN})$ classifier is one of the simplest supervised learning classification algorithms [26]. KNN is a lazy evaluation algorithm; it doesn't use the training set to build a model, but rather keeps the training set to predict the test 


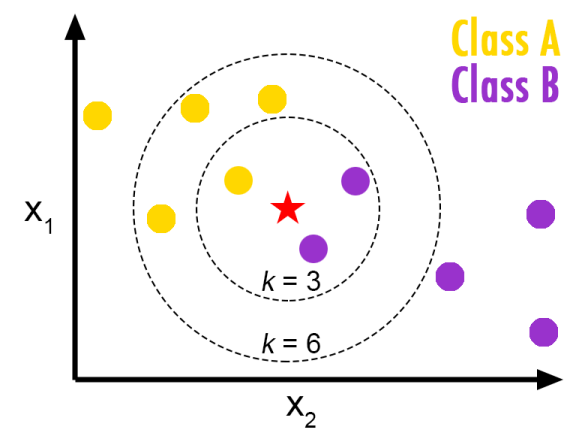

Figure 2.1: $K \mathrm{NN}$ example: The star will be classified by the majority vote of its $k$-nearest neighbors [47]

set. $K \mathrm{NN}$ predicts the class of a point $d$ based on its proximity to points with a known class label. The algorithm works by calculating the distance between point $d$ and every other point in the training set $D$, selecting the $k$ most similar (i.e., closest) points to $d$, and assigning $d$ 's class to be the majority class from the $k$ closest points. As demonstrated in Figure 2.1, different chosen values of $k$ may result in a different classification of a point $d$. The distance (or similarity) between two points can be calculated by multiple distance and similarity measures. Some common distance/similarity measures include:

- Euclidean distance:

$$
d\left(d_{1}, d_{2}\right)=\sqrt{\sum_{i=1}^{n}\left(d_{1}\left[A_{i}\right]-d_{2}\left[A_{i}\right]\right)^{2}}
$$

- Manhattan distance:

$$
d\left(d_{1}, d_{2}\right)=\sum_{i=1}^{n}\left|d_{1}\left[A_{i}\right]-d_{2}\left[A_{i}\right]\right|
$$

- Cosine similarity:

$$
\cos \left(d_{1}, d_{2}\right)=\frac{d_{1} \cdot d_{2}}{\left\|d_{1}\right\| \cdot\left\|d_{2}\right\|}=\frac{\sum_{i=1}^{n} d_{1}\left[A_{i}\right] \cdot d_{2}\left[A_{i}\right]}{\sqrt{\sum_{i=1}^{n} d_{1}\left[A_{i}\right]^{2}} \cdot \sqrt{\sum_{i=1}^{n} d_{2}\left[A_{i}\right]^{2}}}
$$




\subsubsection{Support Vector Machine}

A Support Vector Machine (SVM) is a supervised machine learning algorithm that essentially builds a hyperplane separating two classes in $d$-dimensional feature space [12]. Given a training set $(X, Y)=\left(\bar{x}_{i}, y_{i}\right)$, an SVM attempts to select an optimal hyperplane $h(\bar{x})=\mathbf{w} \cdot \bar{x}+b$, w.r.t. a specialized criterion. The optimal hyperplane will have maximized the distance between the nearest data point from either class of the training set, called the margin. SVMs use the points in the training set that are closest to the hyperplane, called support vectors, to establish the hyperplane equation. The optimization problem of finding the hyperplane that maximizes the margin is represented as:

$$
\min _{\mathbf{w}, b}\left(\frac{\|\mathbf{w}\|^{2}}{2}\right)
$$

subject to constraints:

$$
y_{i}\left(\mathbf{w} \cdot \bar{x}_{i}+b\right) \geq 1, \forall \bar{x}_{i} \in X
$$

Figure 2.2 shows potential hyperplanes and an optimal hyperplane separating two classes.

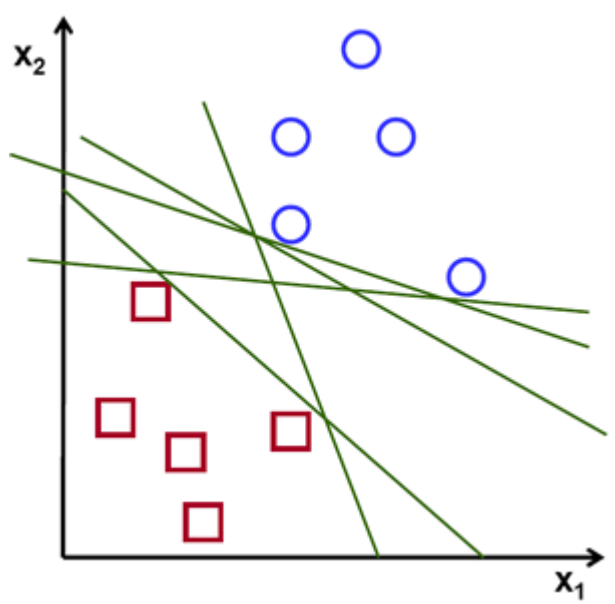

(a) Some potential hyperplanes separating two classes.

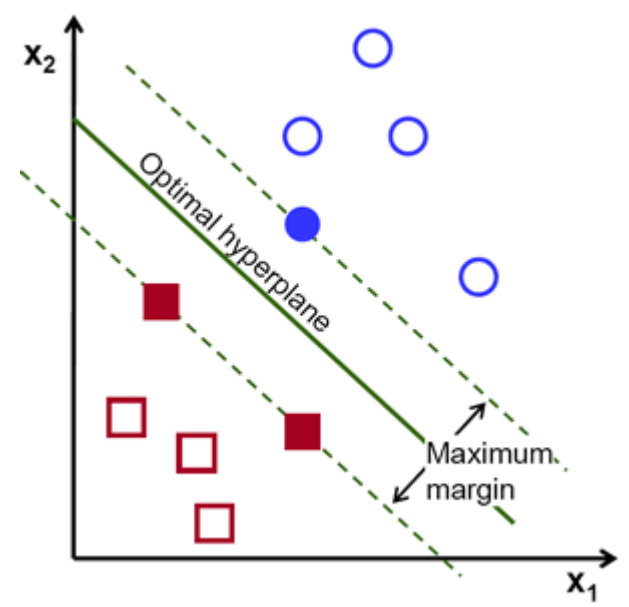

(b) The optimal hyperplane maximizes the margin between two classes.

Figure 2.2: Support Vector Machine hyperplane example [44] 


\subsubsection{Random Forests}

Random Forest is a bagging extension of the Decision Tree classifier [7]. Decision trees are simple and efficient supervised learning classifiers that represent a tree-like model of decisions. The $\mathrm{C} 4.5$ recursive decision tree induction algorithm, proposed by Quinlan in [52], divides the data into smaller and smaller subsets based on chosen attributes until either the subset contains only points with the same class label or there are no more attributes to split the data on, and constructs the tree. The splitting attribute can be selected based on the information gain measure or the information gain ratio, so that the data is split into the purest subsets.

Shown in Algorithm 1, Random Forest builds an ensemble of decision trees, where each decision tree is built from a subset of the training data and a subset of the attributes. The subsets of training data are built from resampling the training set with replacement, while the subsets of attributes are randomly sampled without replacement. By creating decision trees from different subsets of the training data, Random Forests can help prevent the overfitting problem that decision trees sometimes exhibit. In addition, combining the decision trees allows variance to decrease without increasing the bias, which allows for Random Forest to achieve a higher accuracy than decision trees.

\subsubsection{Boosting}

Boosting is an ensemble technique for reducing misclassification error of any given classifier. The main idea of boosting is to sequentially train a set of weak classifiers into a strong one, and in doing so generating an ensemble of classifiers. Each new classifier is built to correct its predecessor's errors by giving higher weights to the misclassified data points in the training set. This way, the new classifier knows which points to focus on. The final classifier is built through weighting the full ensemble's 


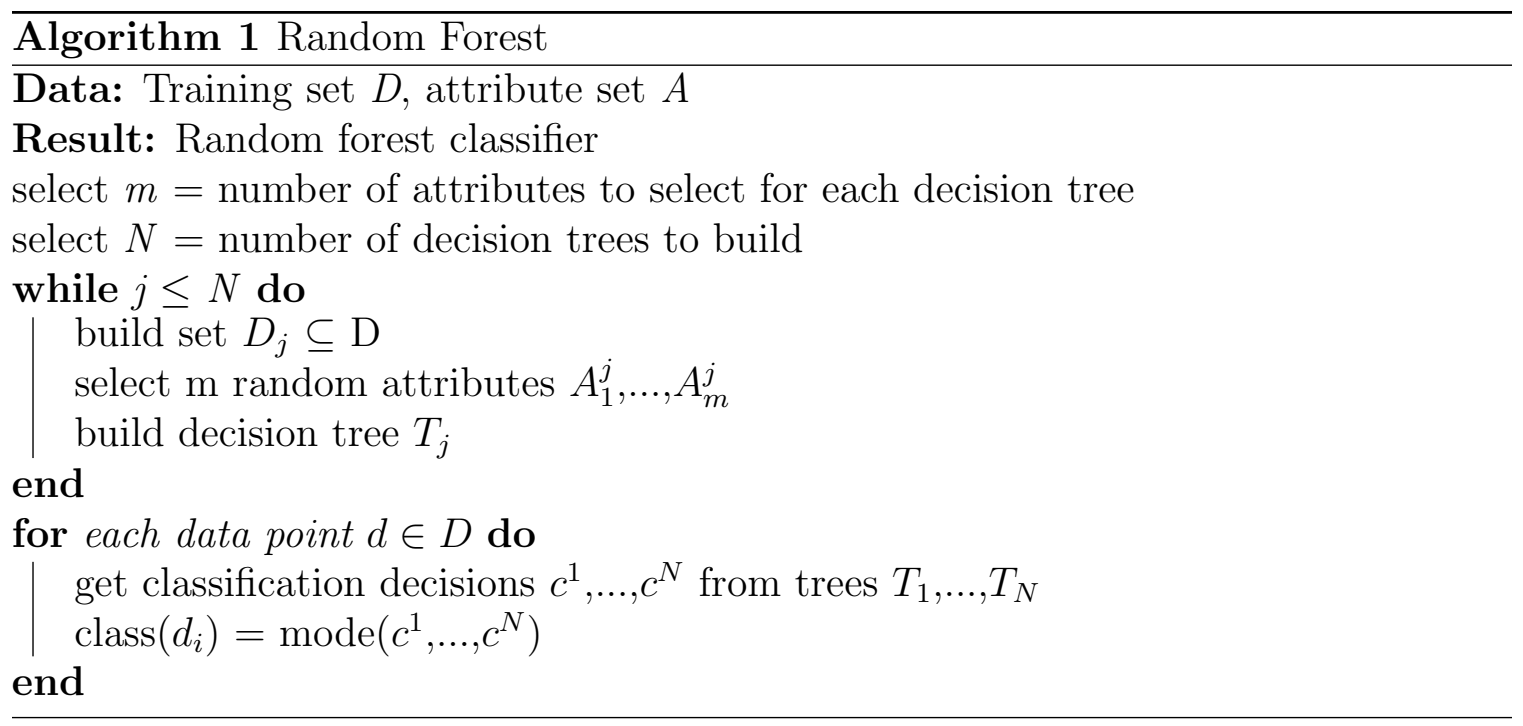

votes by their weighted classification error rate. The classic example of a boosting classifier is AdaBoost, or the Adaptive Boosting algorithm [25]. In our work, we use an extension of the original AdaBoost algorithm, AdaBoost-SAMME [62], which we describe in further detail in Chapter 4.

Similar to AdaBoost, gradient boosting also sequentially trains an ensemble of classifiers, with each new classifier attempting to correct the previous one. The difference between gradient boosting and AdaBoost is that, rather than updating the weights of every misclassified point at every iteration, gradient boosting attempts to train the new classifier with the residual errors made by its predecessor. It gradually minimizes the loss function using gradient descent to find the mistakes in the previous classifier's attempt.

\subsubsection{Hierarchical Classification}

Traditional classification problems involving no inherent class hierarchy are sometimes referred to as flat classification problems. In hierarchical classification problems, the classes are structured in a hierarchy with parent-child relationships between classes. This hierarchical structure can either be a tree or a directly acyclic graph (DAG). In 
this work, we use a tree-based class hierarchy, as each child posture can only belong to one parent posture.

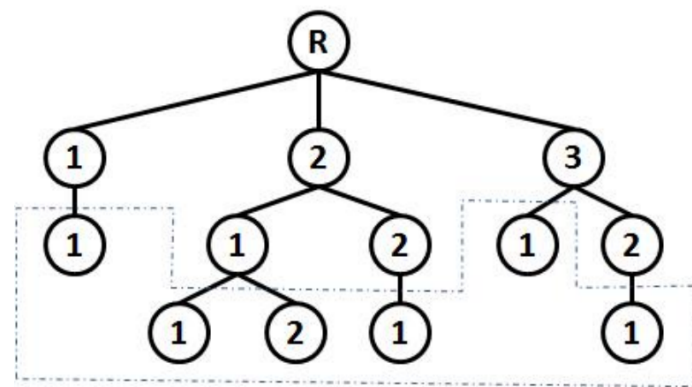

(a) Flat Classification Approach

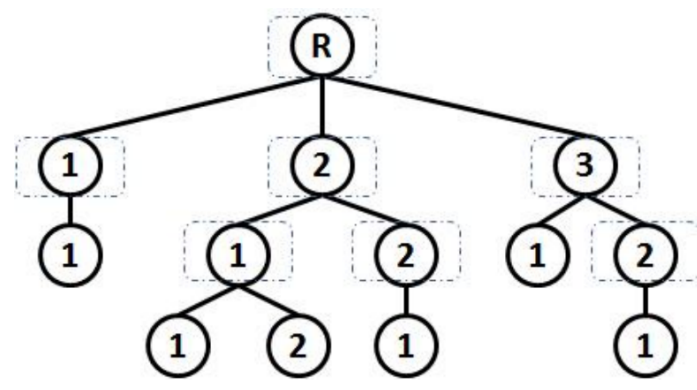

(c) Local Per Parent Node Approach

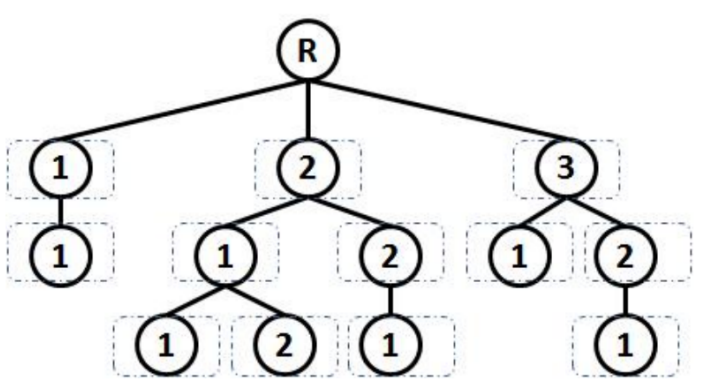

(b) Local Per Node Approach

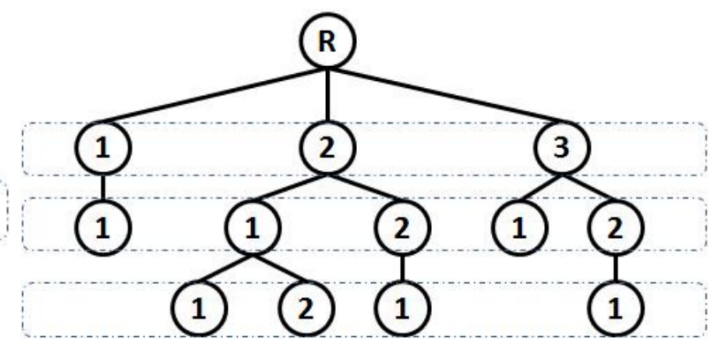

(d) Local Per Level Approach

Figure 2.3: Four types of hierarchical classification approaches [46]

Figure 2.3 shows four types of hierarchical classification approaches, where the dashed squares illustrate classifiers predicting child classes. A simple example of a hierarchical classification problem - classifying fruit - is shown in Figure 2.4. The flat classification approach (Figure 2.3(a)) is the simplest as it predicts only leaf classes and works like a traditional classification algorithm. For instance, in the example illustrated in Figure 2.4, a flat classifier would be predicting the following class labels: "red apple", "cherry", "strawberry", "banana", "lemon", "green apple", "pear", and "green grape". Hierarchical classification algorithms can be categorized into two approaches: local or global. The global approach trains a single model for all of the hierarchical classes. In contrast, local approaches train a hierarchy of models, where one model is associated with each class node and predicts the subclasses of this node. Depending on how the classifiers explore the local hierarchy, local approaches 
fall into three categories: local per node, local per parent node, and local per level [54]. Figure 2.3(b) illustrates the local classifier per node approach, where a single binary classifier is trained on each node of the hierarchy, excluding the root node. This results in a hierarchy of flat classifiers. For the example in Figure 2.4, binary classifiers are trained on all nodes except for the root "Fruit" node. In the local classifier per parent node approach, each parent node has a classifier that is trained to classify its child classes, shown in Figure 2.3(c). Per the example, separate classifiers are trained on "fruit", "red fruit", "medium-sized red fruit", "small red fruit", "yellow fruit", "thin-shaped yellow fruit", "round yellow fruit", "green fruit", "medium-sized green fruit", "small green fruit", "round green fruit", and "non-round green fruit". Finally, the local classifier per level approach trains one flat classifier on each hierarchical

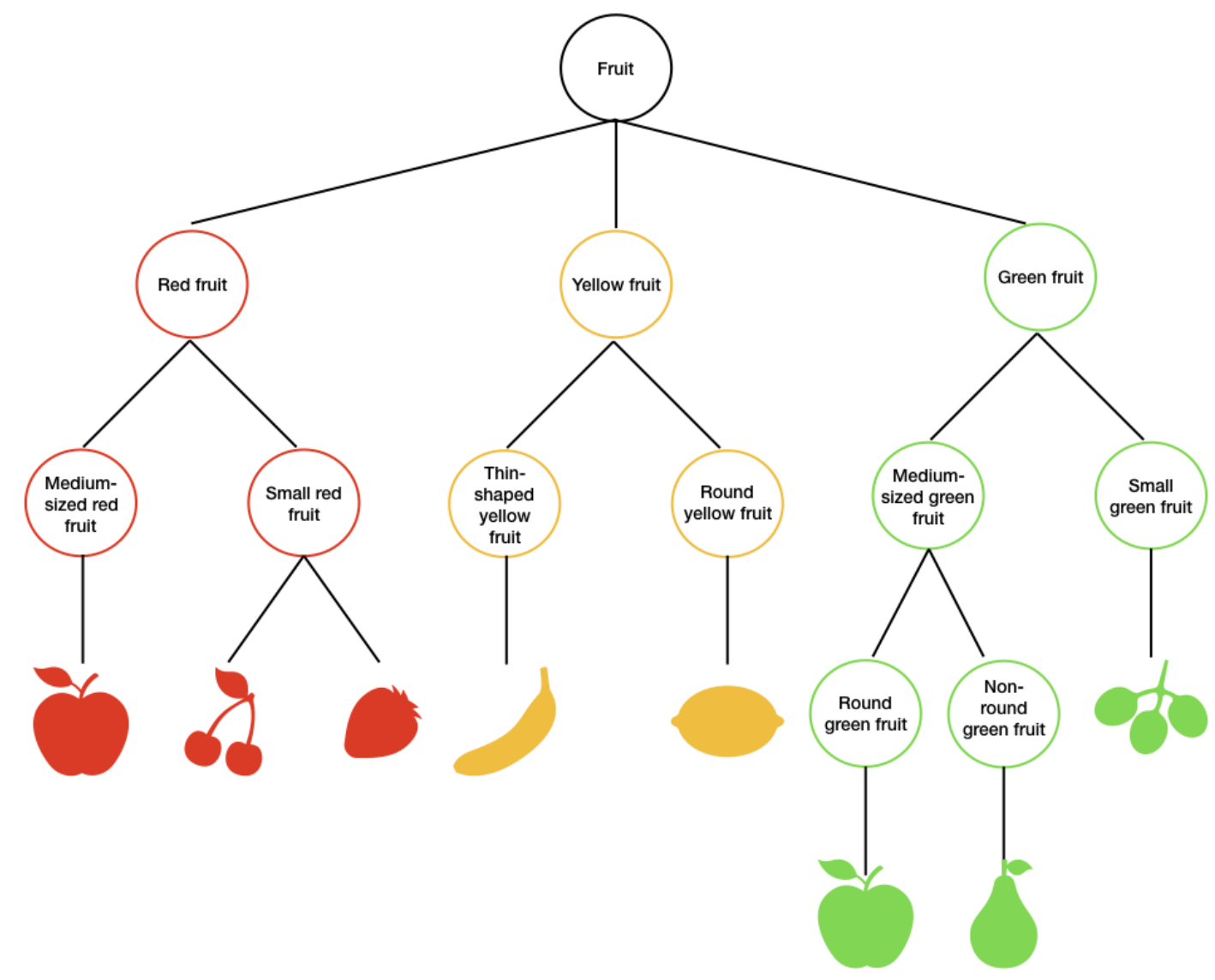

Figure 2.4: Example of a hierarchical classification problem 
level. Figure 2.3(d) illustrates this classifier.

In this work, we use the local classifier per parent node approach on two different hierarchical class structures. 
Chapter 3

\section{RELATED WORK}

Previous studies have validated various monitor types and monitor placements using different data processing methods in controlled laboratory settings. Zhang et al. [61] successfully classified 32 physical activity types using an Intelligent Device for Energy Expenditure and Activity (IDEEA), a new microcomputer-based portable physical activity measurement system consisting of several accelerometers positioned on the chest, thighs, and feet. Bonomi et al.[6] used decision tree models to identify seven activity classes from accelerometers placed on participants' lower backs. Although they were able to obtain a classification accuracy of $93 \%$ using intervals of 6.4 or 12.8 seconds, future work needs to be done in order to validate their models in a free-living setting. Gyllensten et al. [27] trained support vector machine, feed-forward neural network, decision tree, and majority voting models on a waist-mounted accelerometer, and evaluated the reproducibility of the accuracy of laboratory-trained classifiers on real life data. They found that the performance of all four laboratory-trained classification algorithms significantly decreased when using free-living data, with the largest decrease in $F$-score being from $99 \%$ to $55 \%$.

Staudenmayer et al. [56] developed a method using neural networks, SVMs, and random forest to predict energy expenditure, activity intensity, sedentary time, and locomotion time on laboratory data from a wrist-worn ActiGraph monitor. Using 15second windows, their random forest performed the best out of all of their machine learning models, predicting activity intensity with $75 \%$ accuracy, locomotion time with $99 \%$ accuracy, and sedentary time with $96 \%$ accuracy. Although the Staudenmayer method provides evidence that wrist acceleration data can be used to estimate energy expenditure and detect sedentary and locomotion time relatively accurately 
on laboratory data, it doesn't go without its limitations. For instance, they placed the ActiGraph on the dominant wrist rather than non-dominant wrist, which is what is used in the National Health and Nutrition Examination Survey's (NHANES) ActiGraph data analysis, the largest nationally representative database for objectively monitored human physical activity. The data in their study was also collected from a small number of participants in a laboratory setting, which is considered a limitation as there have been studies that show models trained on laboratory collected data do not necessarily perform as well on free-living data [27, 20]. Staudenmayer et al. obtained some promising preliminary results by applying their methods to two participants' free-living wrist accelerometer data, but additional investigation is needed to further validate that their methods work with free-living data. Our work builds on the work in [56] by collecting a larger sample of free-living data from an ActiGraph placed on the non-dominant wrist, and making use of the same seven variables to summarize acceleration signals of 1 second intervals. We also used this series of features to summarize thigh and chest-mounted accelerometer signals.

Ellis et al. [20] classified hip and wrist accelerometer data into four activity classes using a two-layer machine learning method consisting of a random forest and hidden Markov model. In their study, 40 participants were recruited and free-living data was collected over seven consecutive days as a SenseCam wearable camera captured ground truth behavior data. Their hip classifier achieved an average of $89.4 \%$ balanced accuracy over the four activities, and their wrist classifier obtained $84.6 \%$ using one-minute interval windows. Ellis et al. captured their ground truth using a SenseCam which took still images every 20 seconds. In our work, we collect video using a GoPro Hero 5 camera, which allows us to perform more detailed analysis and therefore classify more detailed activity classes than when using the still images from the SenseCam. We set up a similar experiment to [20] to observe how our models perform when trying to predict the same activity classes. 
Dr. Sarah Kozey Keadle of the Cal Poly Kinesiology and Public Health Department collaborated with Cal Poly Data Science Capstone students to develop machine learning models to predict sedentary versus non-sedentary behavior using free-living data from a wrist-worn accelerometer [41]. Their study uses a subset of the same Actigraph wrist monitor data that is used in this thesis, and therefore lays some of the groundwork for our work. The Data Science Capstone work considers 25 people who wore an Actigraph wrist monitor for seven consecutive days and participated in two two-hour direct observation sessions belonging to one out of five activity domains (active leisure, sedentary leisure, household, errands, and work). Cal Poly Kinesiology and Public Health Department research assistants manually coded the ground truth criterion into seven activity classes: active, sitting still, sitting and typing, sitting with upper body movement, lying, kneeling, and private / not coded. Because the ground truth criterion is manually coded via a frame-by-frame analysis of the observation video, only 20 observation sessions amongst 12 participants were coded completely by the time of their investigation. Their most successful model was their random forest classifier, which predicted sedentary vs. non-sedentary behavior with an overall accuracy of $73.98 \%$ using $k$-fold cross validation $(k=5)$ [41]. The Data Science Capstone work demonstrated that models trained on free-living data more accurately predict sedentary behavior at the second level than previous lab-trained models, specifically [56]. The work documented in this thesis uses the completely coded dataset of 25 participants wearing the Actigraph on their wrist, in addition to two BioStamp monitors on their thigh and chest. Using the Data Science Capstone team's project as baseline, we continue and expand on their work by exploring more models and increasing the granularity of the activity types we are predicting. 
Chapter 4

\section{DESIGN AND IMPLEMENTATION}

This work utilizes data collected by the Cal Poly Kinesiology and Public Health Department. We used the raw accelerometer data they collected from three different monitors to create features that describe participants' acceleration per second, and merged these features with the ground truth observation data they provided to construct our training set. We used flat classification algorithms as well as a top-down hierarchical classification approach to predict sedentary versus non-sedentary behavior and type of physical activity to different levels of granularity.

\subsection{Kinesiology Experimental Design}

The data collection took place at the Cal Poly Department of Kinesiology and Public Health. In total, 25 participants between 18-59 years old were recruited and signed informed consent documents. Each participant completed two two-hour sessions over a period of seven days. While they were observed, they wore two BioStamp accelerometers - one on their chest and another on their thigh, and one ActiGraph monitor on their non-dominant wrist. During these direct observation sessions, the research assistants recorded participants with a GoPro Hero 5 camera, which served as the ground truth, as they completed two out of five activity domains that are representative of activities done in daily life with distinct movement patterns: active, household, errands, leisure, and work.

Once data was collected, research assistants performed a frame-by-frame analysis of the observation session video recordings using an event recorder program, Observer XT. Each video was manually coded to identify type of behavior and posture 


\begin{tabular}{|l|l|}
\hline Activity Domain & Description \\
\hline \hline Household & $\begin{array}{l}\text { Household activities or self-care activities for a minimum of 30 min- } \\
\text { utes observed time (e.g., meal prep, clean up) }\end{array}$ \\
\hline Work & $\begin{array}{l}\text { Typical work-related activities; getting up from the chair at least } \\
\text { twice during observation }\end{array}$ \\
\hline $\begin{array}{l}\text { Errands and } \\
\text { Transportation }\end{array}$ & $\begin{array}{l}\text { Behaviors in community (e.g., errands, shopping, attending an } \\
\text { event) Some forms of transportation (car, bus, train, walk, or bike) }\end{array}$ \\
\hline $\begin{array}{l}\text { Sedentary } \\
\text { Leisure }\end{array}$ & $\begin{array}{l}\text { Typical leisure time behaviors; out of work/school or on the week- } \\
\text { end. At some point watching TV/video or playing video games }\end{array}$ \\
\hline Active Leisure & Spending at least 30-45 minutes in exercise or sport \\
\hline
\end{tabular}

Table 4.1: Activity domains and participant directions

according to a multi-pass coding scheme. The behavior coding identifies what the participant is doing, specifically taking into consideration the location and purpose of the activity. Behaviors are coded in accordance with the American Time Use Survey Activity Lexicon [9]. Inter-rater agreement between coders was high (intraclass correlation coefficient $>0.9$ ). Table 4.2 lists the behavior coding options.

Posture was coded to identify body postures, and extra detail was provided classifying upper body movement and intensity. Intensity is coded in terms of metabolic equivalents (METs). METs are the most common unit for measuring activity intensity, and are used to describe the four intensity categories: less than 1.5 METs is categorized as sedentary, between 1.5-2.99 METs is categorized as light intensity, moderate intensity is described as between 3.0-5.99 METs, and activity greater than 6.0 METs is categorized as vigorous intensity [37]. Figure A.3 in Appendix A provides the posture coding options and their associated upper body and intensity options. The frame-by-frame analysis generated the ground truth criterion data that was used to develop the training set. 


\begin{tabular}{|c|c|}
\hline Major Behavior Category & 2nd-Tier Behavior Category \\
\hline Personal Care & $\begin{array}{l}\text { Sleeping } \\
\text { Grooming, Health-related } \\
\text { Other personal care }\end{array}$ \\
\hline Household Activities & $\begin{array}{l}\text { Housework } \\
\text { Food prep and cleanup } \\
\text { Interior maintenance, repair, and decoration } \\
\text { Exterior maintenance, repair, and decoration } \\
\text { Lawn, garden, and houseplants } \\
\text { Animals and pets } \\
\text { Household management/other household activities }\end{array}$ \\
\hline $\begin{array}{l}\text { Caring For and Helping } \\
\text { Household Members }\end{array}$ & $\begin{array}{l}\text { Caring for and helping children } \\
\text { Caring for and helping adults }\end{array}$ \\
\hline $\begin{array}{l}\text { Work and Work-Related } \\
\text { Activities }\end{array}$ & $\begin{array}{l}\text { General } \\
\text { Screen-based }\end{array}$ \\
\hline Education & $\begin{array}{l}\text { Taking class, research, homework } \\
\text { Extracurricular (not sports) }\end{array}$ \\
\hline $\begin{array}{c}\text { Organizational, Civic, and } \\
\text { Religious Activities }\end{array}$ & Organizational civic, volunteer, and religious activities \\
\hline Purchases & Purchasing goods and services \\
\hline Eating and Drinking & Eating and drinking, waiting \\
\hline $\begin{array}{l}\text { Socializing, Relaxing, and } \\
\text { Leisure }\end{array}$ & $\begin{array}{l}\text { Socializing, communicating, non-screen based } \\
\text { Screen-based (TV, video games, computer, phone) }\end{array}$ \\
\hline $\begin{array}{l}\text { Sports, Exercise, and } \\
\text { Recreation }\end{array}$ & $\begin{array}{l}\text { Participating in sport, exercise or recreation } \\
\text { Attending sport, exercise or recreation event, perfor- } \\
\text { mance }\end{array}$ \\
\hline Traveling & $\begin{array}{l}\text { Driver (car/truck/motorcycle) } \\
\text { Passenger (car/truck/motorcycle) } \\
\text { Passenger (bus/train/airplane) } \\
\text { Biking } \\
\text { Walking } \\
\text { General }\end{array}$ \\
\hline
\end{tabular}

Table 4.2: Behavior coding options.

\subsection{Dataset Construction}

The research team provided two datasets: ground truth data and raw accelerometer data for all three monitors that were used to create the training dataset. Developing the proper training set was a non-trivial process. The provided ground truth data described each two-hour direct observation session by the amount of time relative to 


\begin{tabular}{|l|l|l|}
\hline Posture & Upper body Modifier & Intensity Modifier \\
\hline \hline Lying down & $\begin{array}{l}\text { No movement, Unidentifiable, } \\
\text { typing, yes movement }\end{array}$ & Sedentary \\
\hline Sitting/reclining & $\begin{array}{l}\text { No movement, Unidentifiable, } \\
\text { typing, yes movement }\end{array}$ & Sedentary \\
\hline Kneeling/squatting & $\begin{array}{l}\text { No movement, Unidentifiable, } \\
\text { typing, yes movement }\end{array}$ & Sedentary \\
\hline Stretching & Unknown & $\begin{array}{l}\text { Sedentary, light, moderate, } \\
\text { vigorous }\end{array}$ \\
\hline Standing & $\begin{array}{l}\text { No movement, Unidentifiable, } \\
\text { typing, yes movement }\end{array}$ & Light \\
\hline Stand and move & No movement & Light, moderate, vigorous \\
\hline $\begin{array}{l}\text { Stand and move with } \\
\text { upper body movement }\end{array}$ & Yes & Light, moderate, vigorous \\
\hline $\begin{array}{l}\text { Stand and move with } \\
\text { unidentifiable upper } \\
\text { body movement }\end{array}$ & Unknown & Light, moderate, vigorous \\
\hline Walk & Unknown & Light, moderate, vigorous \\
\hline Walk with load & Unknown & Light, moderate, vigorous \\
\hline Running & Unknown & Moderate, vigorous \\
\hline Bike & No & Moderate, vigorous \\
\hline Ascending stairs & Unknown & Moderate, vigorous \\
\hline Descending stairs & Unknown & Unknown \\
\hline Sports & igorous \\
\hline
\end{tabular}

Table 4.3: Posture coding options with their upper body and intensity modifier options.

the start of the observation, the duration of each behavior/posture, the actual behavior/posture of the observed participant, optional upper body movement, sporting activity, posture intensity, and type of work modifiers, and the state of the behavior/posture. Table 4.4 further details the columns provided in the ground truth criterion log; a sample of the ground truth data is provided in Appendix A.

\subsubsection{Ground Truth.}

We reformatted and expanded the ground truth criterion log into the proper format to be merged with the monitor data using R. Each row now serves as one second of 


\begin{tabular}{|l|l|}
\hline Column Name & Description \\
\hline \hline Date_Time_Absolute_dmy_hmsf & Date and time the observation was coded \\
\hline Date_dmy & Date, month, and year the observation was coded \\
\hline Time_Absolute_hms & $\begin{array}{l}\text { Time in hours, minutes, and seconds the observa- } \\
\text { tion was coded }\end{array}$ \\
\hline Time_Absolute_f & $\begin{array}{l}\text { Fraction of a second of the time the observation } \\
\text { was coded }\end{array}$ \\
\hline Time_Relative_hmsf & $\begin{array}{l}\text { Time relative to the start of the observation in } \\
\text { hours, minutes, seconds, and fraction of a second }\end{array}$ \\
\hline Time_Relative_hms & $\begin{array}{l}\text { Time relative to the start of the observation in } \\
\text { hours, minutes, and seconds }\end{array}$ \\
\hline Time_Relative_sf & $\begin{array}{l}\text { Time in seconds and fraction thereof relative to } \\
\text { the start of the observation }\end{array}$ \\
\hline Duration_sf & $\begin{array}{l}\text { Duration in seconds and fraction thereof the be- } \\
\text { havior was performed for }\end{array}$ \\
\hline Observation & Name of the observation session \\
\hline Event log & Event log \\
\hline Behavior & Behavior or posture coding \\
\hline Modifier_1 & $\begin{array}{l}\text { Upper body modifier (Yes movement, no move- } \\
\text { ment, unidentifiable, typing) }\end{array}$ \\
\hline Modifier_2 & Sport modifier (Type of sport being played) \\
\hline Modifier_3 & $\begin{array}{l}\text { Intensity modifier (Sedentary, light, moderate, vig- } \\
\text { orous) }\end{array}$ \\
\hline Modifier_4 & Work modifier (Type of work) \\
\hline Event_Type & $\begin{array}{l}\text { State of the behavior/posture being observed (i.e., } \\
\text { state start, state stop, state point) } \\
\text { Additional comments }\end{array}$ \\
\hline Comment &
\end{tabular}

Table 4.4: Columns in the provided ground truth criterion log.

observation that is labelled with a paricular behavior/posture. The actual date and time of the observation session is acquired from the direct observation timestamp log provided by the research team, presented in Appendix A. Because postures change within and across behaviors, and behaviors can also change during the same and across different postures, we split the original Behavior column into two columns to separate behaviors and postures. The original Modifier_1 and Modifier_3 columns are specific to posture, representing upper body movement and intensity level and we relabelled them as such. We populated any blank cells in these columns with the appropriate default value for the associated posture (i.e., "unknown" for upper body, 


\begin{tabular}{|l|l|}
\hline Column & Description \\
\hline \hline Observation & Name of the observation session \\
\hline Date & Date of the observation session \\
\hline Coding & Sedentary/non-sedentary behavior coding \\
\hline Primary_behavior & Behavior observed for at least half of the current second \\
\hline Primary_posture & Posture observed for at least half of the current second \\
\hline Primary_upper body & Upper body modifier for the primary_posture \\
\hline Primary_intensity & Intensity level of the primary_posture \\
\hline Secondary_behavior & Behavior observed for less than half of the current second \\
\hline Secondary_posture & Posture observed for less than half of the current second \\
\hline $\begin{array}{l}\text { Secondary_upper } \\
\text { body }\end{array}$ & Upper body modifier for the secondary_posture \\
\hline Secondary_intensity & Intensity level of the secondary_posture \\
\hline Num_postures & Number of total postures observed within the current second \\
\hline Transition & $\begin{array}{l}1 \text { if the second contains a transition between postures, other- } \\
\text { wise } 0\end{array}$ \\
\hline Actual_time & Time of the observation session \\
\hline Time & Time relative to the start of the observation session, in seconds \\
\hline
\end{tabular}

Table 4.5: Columns in the second-by-second ground truth files.

the posture's lowest intensity level as shown in Appendix A, Figure A.3). The original Modifier_2 column represents the type of sporting activity, and is only associated with the "EX-participating in sport, exercise or recreation" behavior. For the final ground truth data we absorbed the type of sporting activity into the name of the behavior. For example, if the original provided ground truth criterion had a "EXparticipating in sport, exercise or recreation" as the Behavior and "jogging" as its Modifier_2, the final ground truth data represents this as "EX-jogging". Modifier_4 represents work type (i.e., "Education and Health Services" or "Office (business, professional services, finance, info)"). It is only associated with the two working behaviors ("WRK- general", "WRK- screen based") and is handled similarly to the sport modifier.

In the provided ground truth data, behavior and posture changes were coded up to the hundred-thousandths of a second; therefore, one second could be split by more than one behavior and/or more than one posture. In order to properly 
demonstrate this, the final ground truth data contains two sets of behavior/posture codings: primary and secondary. The primary coding represents the behavior and/or posture that is maintained for more than $50 \%$ of the second, and the secondary coding represents the behavior and/or posture for the remainder of the second. However, if a behavior and/or posture is maintained for at least $80 \%$ of the second, it is considered as the majority coding. In this case, the primary coding is this majority coding, and there is no secondary coding. We created a transition column, where seconds are labelled 1 if they contain a transition between more than one posture, and 0 otherwise. The number of postures contained in each second is also recorded. Finally, in order to directly compare model performance with the methods in [56], we added an extra coding column to label the posture as either a "sedentary" or "non-sedentary" behavior. The outputs of this data transformation are second-by-second ground truth files for each individual participant's direct observation sessions. Table 4.5 lists the columns of the second-by-second ground truth files.

\subsubsection{Raw Accelerometer Data.}

We ran the provided raw accelerometer data through another $\mathrm{R}$ script based on code from [56] to produce aggregated data for each participant. We generalized the $\mathrm{R}$ code to account for the different sampling frequencies of the different monitors, as the ActiGraph has a sampling rate of $80 \mathrm{Hertz}(\mathrm{Hz})$, while the BioStamps have a 31.25 $\mathrm{Hz}$ sampling rate. The raw accelerometer data from the Actigraph contained seven days' worth of data for each participant, totalling over 48 million acceleration samples per data file. An example of a partipant's raw data file is shown in Figure 4.1.

We modified the $\mathrm{R}$ code from from [56] to generate aggregated features for every 80 acceleration samples. One second time intervals were chosen because they are a small enough epoch length to observe transitions between behaviors, and further 
granularity is not physiologically meaningful and difficult to code. We identified seven features to describe the movement the monitor experienced each second. To be directly comparable to the work documented in [56], the same series of features were used: mean vector magnitude, standard deviation vector magnitude, mean acceleration angle, standard deviation acceleration angle, percentage of power between 0.6 and $2.5 \mathrm{~Hz}$, dominant frequency (from the Fourier transform), and the fraction of the dominant frequency over all others. We also created 16 additional features as an attempt to provide more information about the acceleration signal. These additional features are listed in Table 4.6. In total, we aggregated 23 total features for each second, generating second-by-second files that span the seven consecutive days the participant wore the ActiGraph monitor.

Because the ground truth only spans two two-hour segments of these seven days, only the aggregated features from the seconds that took place during the direct observation sessions were needed. Using Python, we merged the ground truth for each participant's two hour direct observation session with the aggregated data.

The raw data from the BioStamp accelerometers was provided in a slightly dif-

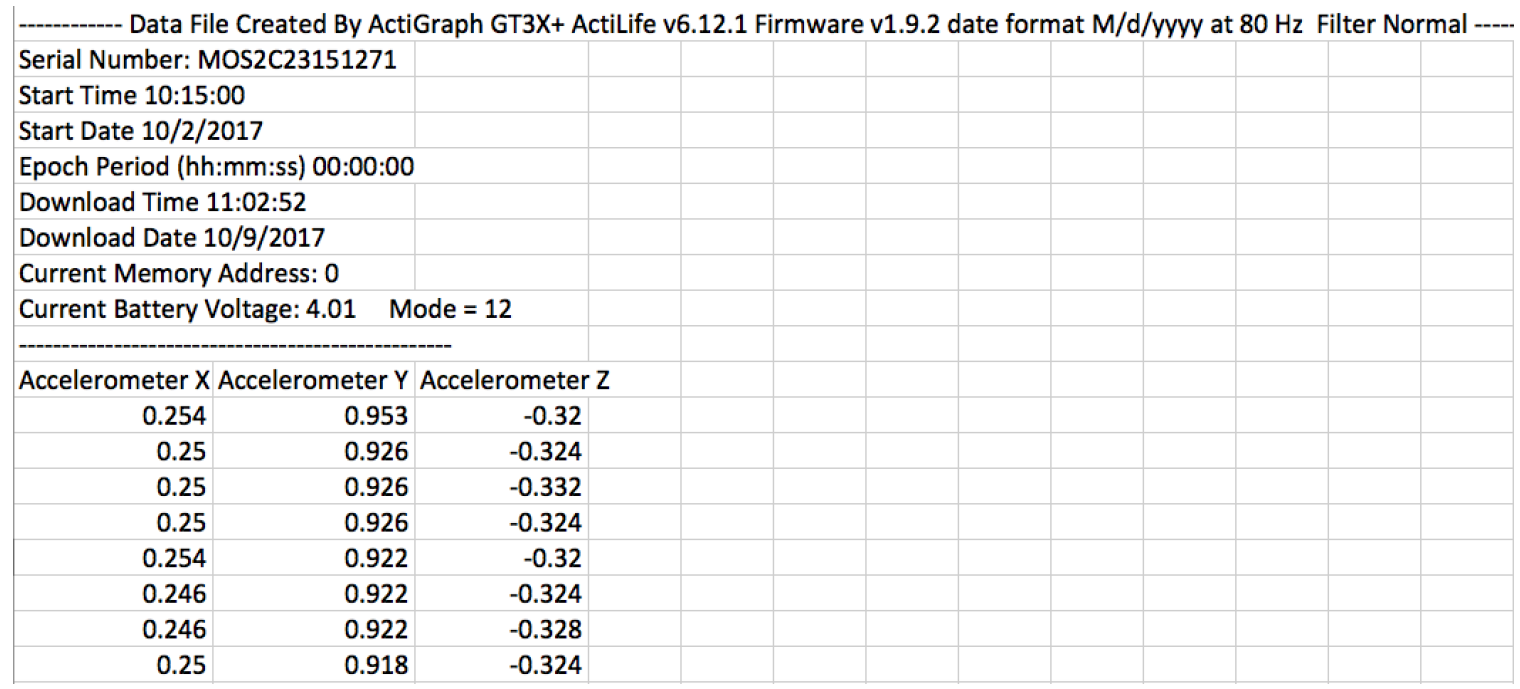

Figure 4.1: An example of the raw data from the Actigraph wrist monitor. 
ferent format, illustrated in Figure 4.2. As previously mentioned, the BioStamps have a $31.25 \mathrm{~Hz}$ sampling rate, meaning each second had about 31 samples in the raw data file. Because the monitors did not necessarily start collecting data at the exact start of a second, the number of samples per second range between 29 and 32 samples. In order to create second-by-second features, we looped through each data file and labelled each sample with the second it belonged to, using the Unix Epoch timestamp provided in the first column. The BioStamp data files were also stored in nested directories, so we modified the $\mathrm{R}$ code to traverse the directory structure.

Unlike the Actigraph, the BioStamps were only worn for a duration of two hours per observation. However, the data for the thigh and chest monitors would start and end at different times for the same observation, therefore the raw thigh and chest data were not exactly synchronized in time. In order to make these monitors comparable to one another and to the Actigraph, we sliced the raw data so that only the seconds that were directly observed according to the timestamp log (Appendix A) were used

\begin{tabular}{|l|l|}
\hline Statistical feature & Definition \\
\hline \hline $\operatorname{Min}(x)$ & Minimum $x$ acceleration in interval \\
\hline $\operatorname{Min}(y)$ & Minimum $y$ acceleration in interval \\
\hline $\operatorname{Min}(z)$ & Minimum $z$ acceleration in interval \\
\hline $\operatorname{Max}(x)$ & Maximum $x$ acceleration in interval \\
\hline $\operatorname{Max}(y)$ & Maximum $y$ acceleration in interval \\
\hline $\operatorname{Max}(z)$ & Maximum $z$ acceleration in interval \\
\hline $\operatorname{Mean}(x)$ & Mean $x$ acceleration in interval \\
\hline $\operatorname{Mean}(y)$ & Mean $y$ acceleration in interval \\
\hline $\operatorname{Mean}(z)$ & Mean $z$ acceleration in interval \\
\hline $\operatorname{SD}(x)$ & Standard deviation of $x$ acceleration in interval \\
\hline $\operatorname{SD}(y)$ & Standard deviation of $y$ acceleration in interval \\
\hline $\operatorname{SD}(z)$ & Standard deviation of $z$ acceleration in interval \\
\hline $\operatorname{Mean}(x * y)$ & Mean $x * y$ acceleration in interval \\
\hline $\operatorname{Mean}(y * z)$ & Mean $y * z$ acceleration in interval \\
\hline $\operatorname{Mean}(x * z)$ & Mean $x * z$ acceleration in interval \\
\hline $\operatorname{Mean}(x * y * z)$ & Mean $x * y * z$ acceleration in interval \\
\hline
\end{tabular}

Table 4.6: Additional statistical features used to summarize the accelerometer signals. 
to create features. Once the appropriate data samples were obtained, we identified features to describe the acceleration in each second - the same 23 features used for the Actigraph. The thigh and chest features were merged by the second using Python, thus creating second-by-second files describing thigh and chest movement for each participant's observation session.

\subsubsection{Merging Ground Truth with Features.}

The final portion of our data construction pipeline combines the aggregated features with the ground truth. Using Python, we extracted the two-hour observation sessions from the aggregated Actigraph features and matched the features with the ground truth observation data for that session. During the merge, we also added a column that contained the activity domain type and participant ID number, so that we could perform leave-one-out cross validation as well as evaluate model performance by activity domain. We created the complete Actigraph dataset by appending all of the observation sessions together.

Merging the aggregated BioStamp features with the ground truth was slightly

\begin{tabular}{|r|r|r|r|}
\hline Timestamp (ms) & Accel X (g) & Accel $Y(g)$ & Accel $Z(g)$ \\
\hline $1.50708 \mathrm{E}+12$ & 0.22437 & 0.7234 & 0.63551 \\
\hline $1.50708 \mathrm{E}+12$ & 0.21509 & 0.73219 & 0.64039 \\
\hline $1.50708 \mathrm{E}+12$ & 0.21314 & 0.76246 & 0.60865 \\
\hline $1.50708 \mathrm{E}+12$ & 0.20288 & 0.78248 & 0.62281 \\
\hline $1.50708 \mathrm{E}+12$ & 0.21704 & 0.78541 & 0.61891 \\
\hline $1.50708 \mathrm{E}+12$ & 0.22681 & 0.77418 & 0.62037 \\
\hline $1.50708 \mathrm{E}+12$ & 0.19507 & 0.75319 & 0.62184 \\
\hline $1.50708 \mathrm{E}+12$ & 0.198 & 0.74879 & 0.62574 \\
\hline $1.50708 \mathrm{E}+12$ & 0.19898 & 0.74879 & 0.62281 \\
\hline $1.50708 \mathrm{E}+12$ & 0.1897 & 0.75758 & 0.62184 \\
\hline $1.50708 \mathrm{E}+12$ & 0.18238 & 0.78639 & 0.63014 \\
\hline $1.50708 \mathrm{E}+12$ & 0.17847 & 0.76979 & 0.62428 \\
\hline $1.50708 \mathrm{E}+12$ & 0.16187 & 0.77711 & 0.63111 \\
\hline & 0.16529 & 0.77565 & 0.6277 \\
\hline
\end{tabular}

Figure 4.2: An example of the raw data from the BioStamp chest monitor. 


\begin{tabular}{|l|l|}
\hline New Posture Coding & Description/Original Postures \\
\hline \hline Lying down & Lying down \\
\hline Sitting & Sitting, sedentary kneeling/squatting, sedentary stretching \\
\hline Standing & Standing \\
\hline Stand and move light & $\begin{array}{l}\text { Stand and move, stand and move with upper body movement, } \\
\text { stand and move with unidentifiable upper body movement at } \\
\text { light intensity }\end{array}$ \\
\hline $\begin{array}{l}\text { Stand and move mod- } \\
\text { erate }\end{array}$ & $\begin{array}{l}\text { Stand and move, stand and move with upper body movement, } \\
\text { stand and move with unidentifiable upper body movement at } \\
\text { moderate intensity }\end{array}$ \\
\hline $\begin{array}{l}\text { Stand and move vigor- } \\
\text { ous }\end{array}$ & $\begin{array}{l}\text { Stand and move, stand and move with upper body movement, } \\
\text { stand and move with unidentifiable upper body movement at } \\
\text { vigorous intensity }\end{array}$ \\
\hline Walk light & Walk, walk with load at light intensity \\
\hline Walk moderate & Walk, walk with load at moderate intensity \\
\hline Walk vigorous & Walk, walk with load at vigorous intensity \\
\hline Running & Running \\
\hline Bike & Bike \\
\hline Ascending stairs & Ascending stairs \\
\hline Descending stairs & Descending stairs \\
\hline Sports & Sports \\
\hline
\end{tabular}

Table 4.7: The new posture coding scheme.

simpler than the Actigraph. Since the aggregated data only spanned the duration of the observation session, we simply had to add a column with the activity domain type and participant ID number, and then merge the aggregated features with the ground truth to create the complete BioStamp dataset.

\subsubsection{Final Dataset.}

We deemed the granularity level of the provided ground truth data logs posture codings too specific for the purpose of this work. Using Python, we constructed a new posture coding column that represents the types of postures we are interested in predicting. This new posture coding scheme is presented in Table 4.7, and is referred to as the full coding scheme in this work. 


\subsection{Questions}

This thesis aims to predict an individual's physical activity/posture based on data from a wrist, thigh, and chest-worn monitor, specifically answering the following questions:

Question 1. What monitor placement and machine learning method best determines sedentary vs. non-sedentary behavior? Prolonged periods of sedentary behavior has been shown to negatively influence metabolic health[45]. Dr. Keadle is interested in understanding the effect of sedentary time on health.

Question 2. How do our sedentary vs. non-sedentary models compare to previous methods? Staudenmayer et. al [56] used laboratory-collected data from a wrist monitor to predict energy expenditure, activity intensity level, sedentary behavior, and locomotion time. We compare the classification accuracies of our models with the random forest model from [56] predicting sedentary vs. non-sedentary behavior.

Question 3. What monitor placement and machine learning method best estimates activity intensity level? Previous investigations [37, 56] have developed methods to estimate minutes in different activity intensities. We build predictive models to determine activity intensity from the three monitors.

Question 4. What monitor placement and classifier best predicts posture into 5 general posture classes? Ellis et. al [20] developed a method to classify free-living behaviors into four behavior labels. While the ground truth coded in our work codes postures into 14 labels, we generalized these labels to five categories in order to observe how our models perform in comparison to the results in [20]. 
Question 5. What monitor placement and machine learning model best predicts posture/intensity into 14 posture classes? Dr. Keadle is interested in developing models to predict activity type at a more granular level. In this work, we generated training models to classify 14 activity labels.

Question 6. Does a hierarchical random forest ensemble improve classification accuracy for predicting 14 posture classes? The 14 class labels of our ground truth can be hierarchically structured. As an attempt to gain lift from our random forest classifiers, we built an ensemble of random forest classifiers to hierarchically classify 14 posture labels.

Question 7. Does using a confusion matrix boosting method improve classification accuracy for predicting 14 posture classes? Given the imbalanced nature of our dataset with respect to the 14 posture classes, we implemented Koço and Capponi's confusion matrix boosting algorithm [34] using Python to overcome the classimbalance problem.

\subsection{Experiments}

In order to answer our questions, we tested a battery of machine learning models on our monitor data using different coding schemes as class variables. The purpose of the different coding schemes, listed in Table 4.8, are to have different levels of granularity in terms of the number of class labels. This allows us to observe model performance as we increase the number of classes we are trying to predict, compare against previous work, and answer our research questions. For our non-binary classifiers, we omitted transition seconds - seconds of which more than one posture was observed - from our dataset. This provides us with "purer" data that happens to be consistent with the experimental circumstances in previous work [20]. Because there are less transitions 
between sedentary to non-sedentary behaviors than there are for our other coding schemes, we did not feel the need to omit transition seconds for our binary classifiers.

\begin{tabular}{|l|l|l|}
\hline Coding Scheme & \# Classes & Description/Postures \\
\hline \hline Sedentary/Non-sedentary & 2 & Sedentary, non-sedentary \\
\hline METs & 4 & Sedentary, light, moderate, vigorous \\
\hline General postures & 5 & $\begin{array}{l}\text { Sit, stand, walk/run, riding in vehicle, } \\
\text { other }\end{array}$ \\
\hline Full coding scheme & 14 & See Table 4.7 \\
\hline
\end{tabular}

Table 4.8: Different coding schemes.

\subsection{1 $\quad K$-Nearest Neighbors.}

We tested multiple values of $k$ for our $k$-nearest neighbors model. In [41], $K=15$ was shown to maximize testing accuracy, so we built a set of models with this value. We then continuously increased $k$ and found that $k=100$ was the best value for all monitors on all coding schemes except for the wrist monitor on the sedentary vs. non-sedentary coding scheme, which performed best when $k=5$. Uniform weights were used (all points in the neighborhood are given equal weights) in both cases.

\subsubsection{Support Vector Machine.}

We used scikit-learn's Linear Support Vector Classifier (LinearSVC) with the maximum number of iterations set to 100 for our SVM model. We chose LinearSVC over SVC because SVC would terminate early without converging to a solution; LinearSVC tends to converge faster than SVC as the number of samples increases. It's important to note that although we limited the maximum number of iterations being 100 for the sake of time, our SVMs still failed to converge. Therefore, the accuracies achieved by these models do not necessarily reflect the true performance of SVMs on this data. 


\subsubsection{Random Forest.}

We tested different sets hyperparameters when building our random forest classifier, varying the maximum depth of the tree, the number of trees in the forest, and the minimum number of samples required to be at a leaf node. While we found that increasing all of these parameters resulted in better performance, we observed the largest improvement when varying the maximum tree depth. All other parameters fell to their default values. We observed that the best value for maximum tree depth was dependent on the monitor and test case. A maximum tree depth of 5 was used in [41] on a subset of our data. We found that this value maximizes testing accuracy for our sedentary vs. non-sedentary coding scheme. However, for our more granular coding schemes, our random forests achieved better performance using a maximum depth of 15 .

\subsubsection{AdaBoost.}

We ran scikit-learn's AdaBoost classifier on our monitor data using 100 random forests. Scikit-learn's AdaBoost classifier implements the SAMME (Stagewise Additive Modeling using a Multi-class Exponential loss function) algorithm, which is an extension of the original AdaBoost algorithm [25] to the multi-class setting without reducing the problem into multiple two-class problems [62].

\subsubsection{Gradient Boosting.}

Since gradient boosting tends to be resilient to over-fitting, we chose to perform 200 boosting iterations. The number of nodes in the tree were limited to 7. Our gradient boosting model has these properties for our results comparison. 


\subsubsection{Hierarchical Classifier.}

We observed that our single-classifier models were only predicting 6 out of 14 classes out of the full coding scheme: sitting, standing, stand and move light, walk moderate, running, and biking. The other intensities of the stand and move and walking postures were not learned and therefore not distinguished from each other. We considered two explanations for this phenomenon. One explanation is that in presence of other data, the differences between certain postures become harder for our models to learn. Therefore, we attempted to improve classification accuracy by structuring our posture classes in a hierarchy and training classifiers to distinguish between more specific postures. A second reason would be that our models are not able to confidently learn the difference between intensities in addition to types of postures because they are learning from an imbalanced dataset.

As an attempt to improve the performance of our single-classifier models, we built an ensemble of classifiers using the local classifier per parent node approach for hierarchical classification to predict the full coding scheme. For this classifier, we structured the postures into a 3-level class hierarchy, shown in Figure 4.3.

Our ensemble consists of five random forest classifiers, one per parent node. The root classifier predicts six postures - lying down, sitting, standing, locomotion, biking,

and other sport. A standing classifier which is trained on only standing and stand and move data, predicts stand or stand and move from the points the root classifier labelled as standing. Another classifier is trained on locomotion data, which includes observations that were coded as walking, ascending stairs, descending stairs, and running. This locomotion classifier predicts these child classes from the data points the root classifier labelled as locomotion. We use the last two classifiers to predict the final level of the hierarchy. The stand and move points labelled by the standing classifier are used by one classifier, which was trained on stand and move data. This 


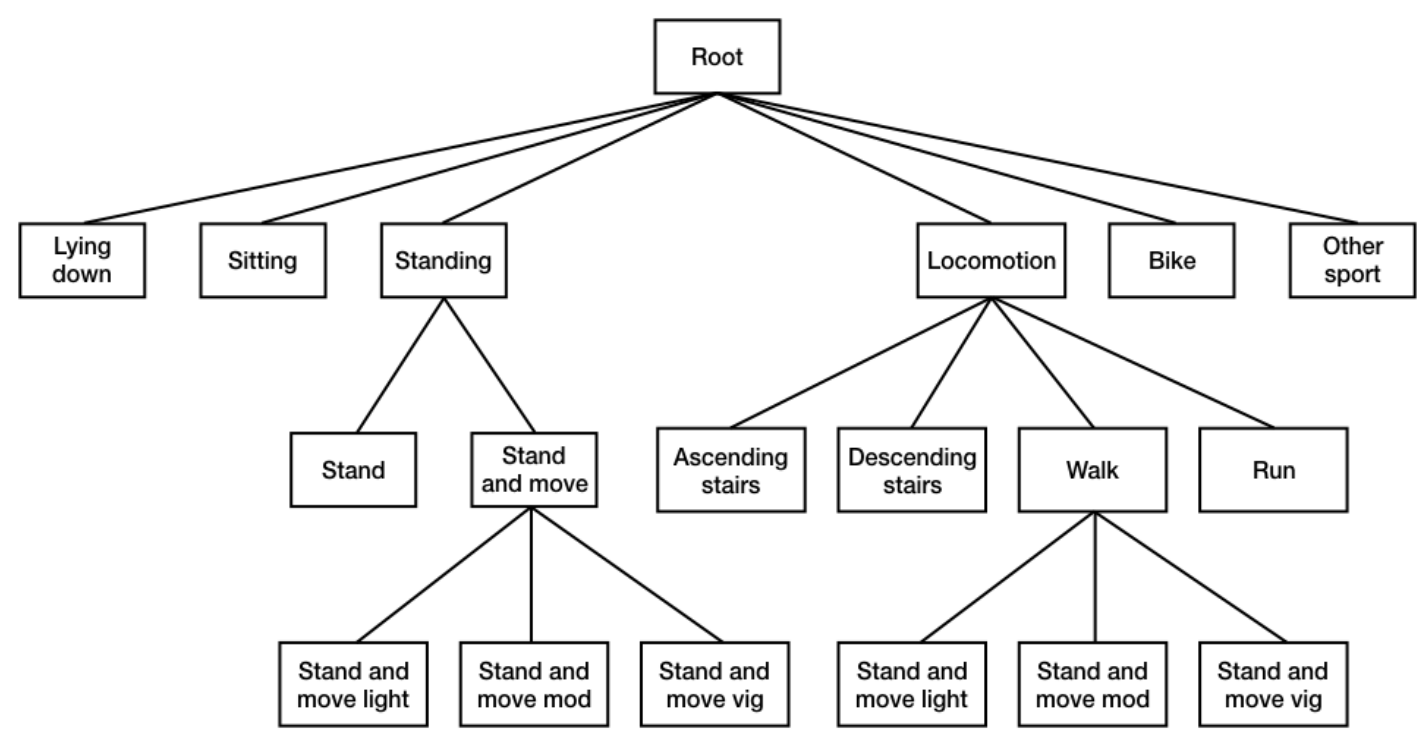

Figure 4.3: Our 3-tier class hierarchy.

stand and move intensity classifier is used to distinguish between the three stand and move intensity levels and predicts stand and move light, stand and move moderate, and stand and move vigorous. Finally the last classifier, trained on purely walking data (i.e., observations that were only coded as walking), predicts the level of walking intensity from the walking points labelled by the walking classifier.

\subsubsection{Confusion Matrix Boosting (CoMBo).}

Our dataset has an imbalance ratio of 0.000017 , from having only 3 other sport movement observations versus 171,985 sitting observation seconds. We implemented the Confusion Matrix BOosting algorithm (CoMBo) [34], an algorithm proposed by Koço and Capponi which extends the multi-class boosting method AdaBoost.MM [42] to 
the imbalanced class problem via greedily minimizing the empirical norm of the confusion matrix. The confusion matrix is one of the most informative performance measures for machine learning classification. It contains the different combinations of predicted versus actual values, and demonstrates the classifier's "confusion" among classes by showing how accurate the classifier is at predicting a certain class, as well as how the classifier tends to misclassify ("confuse") a certain class for another. In order to learn classifiers for the imbalanced class problem, Koço and Capponi [34] propose a framework that makes use of the confusion matrix as an error measure.

In [34], the probabilistic confusion matrix is used overcome the class-imbalance problem. The probabilistic confusion matrix is computed from the raw confusion matrix (i.e., what we know as the standard confusion matrix, where the values of each row sum up to the number of samples of the class of the respective row). In the probabilistic confusion matrix, the values of a row add up to 1 . With this property, Koço and Capponi [34] propose that directly minimizing the confusion matrix norm aids in smoothing the accuracy among imbalanced classes. They introduce an algorithm that minimizes the norm of the confusion matrix, CoMBo, which is shown in Algorithm 2. Let $S$ be a training sample, $T$ be the total number of iterations, and $W$ be a weak learner. The score functions $f$ are initialized to zero and the cost matrix D is initialized so that classes with a larger number of samples are assigned a smaller misclassification cost than classes with less samples. For each training iteration, the weak learner $W$ is used to build the weak classifier $h_{t}$ using the cost matrix $\boldsymbol{D}_{\boldsymbol{t}}$, which is also used to compute the weight $\alpha_{t}$ for $h_{t}$. Also referred to as the importance of $h_{t}, \alpha_{t}$ depends on the edge $\delta_{t}$ which measures the difference between the performance of classifier $h_{t}$ versus random guessing. The cost matrix $\boldsymbol{D}_{\boldsymbol{t}}$ is updated so that misclassified points are given a higher cost than correctly classified points - similarly to how in regular AdaBoost, the misclassified points are given a higher weight. In addition to the ability to correctly predict more difficult samples, the misclassification 
costs are also dependent on the number of samples having the same class $y_{i}$, which is represented by the term $1 / m_{y_{i}}$. Finally, the output prediction is the simple weighted majority vote of all $T$ weak classifiers.

As the implementation of this algorithm isn't publicly available, we implemented the CoMBo algorithm according to the pseudo-code in Algorithm 2. Because our dataset is significantly larger compared to those run in [34] (the largest dataset they tested on was not even $20 \%$ the size of our monitor data), we split our dataset by activity domain and trained on each activity domain separately.

\section{Algorithm 2 CoMBo: Confusion Matrix BOosting}

\section{Given}

- $S=\left\{\left(x_{1}, y_{1}\right), \ldots,\left(x_{m}, y_{m}\right)\right\}$ where $x_{i} \in X, y_{i} \in 1, \ldots, K$

- $T$ : number of iterations, $W$ : weak learner

- $\forall i \in\{1, \ldots, m\}, \forall l \in\{1, \ldots, K\}, f_{1}(i, l)=0$

- $\boldsymbol{D}_{\mathbf{1}}(i, l)= \begin{cases}\frac{1}{m_{y_{i}}} & \text { if } y_{i} \neq l \\ \frac{-(K-1)}{m_{y_{i}}} & \text { if } y_{i}=l\end{cases}$

for $t=1$ to $T$ do

Use $W$ to learn $h_{t}$ with edge $\delta_{t}$ on $\boldsymbol{D}_{\boldsymbol{t}}$, and $\alpha_{t}=\frac{1}{2} \ln \frac{1+\delta_{t}}{1-\delta_{t}}$ where:

$$
\delta_{t}=\frac{-\sum_{i=1}^{m}\left(\boldsymbol{D}_{\boldsymbol{t}}\left(i, h_{t}\left(x_{i}\right)\right)\right.}{\sum_{i=1}^{m} \sum_{l \neq y_{i}}\left(\boldsymbol{D}_{\boldsymbol{t}}(i, l)\right)}
$$

Update D:

$$
\boldsymbol{D}_{\boldsymbol{t + 1}}(i, l)= \begin{cases}\frac{1}{m_{y_{i}}} \exp \left(f_{t+1}(i, l)-f_{t+1}\left(i, y_{i}\right)\right) & \text { if } l \neq y_{i} \\ -\frac{1}{m_{y_{i}}} \sum_{j \neq y_{i}}^{k} \exp \left(f_{t+1}(i, j)-f_{t+1}\left(i, y_{i}\right)\right. & \text { if } l=y_{i}\end{cases}
$$

end for

where $f_{t+1}(i, l)=\sum_{z=1}^{t} \mathrm{II}\left[h_{z}(i)=l\right] \alpha_{z}$

Output final hypothesis:

$$
H(x)=\underset{l \in 1, \ldots, k}{\operatorname{argmax}} f_{T}(x, l), \text { wheref }_{T}(x, l)=\sum_{t=1}^{T} \mathrm{II}\left[h_{t}(x)=l\right] \alpha_{t}
$$


Chapter 5

RESULTS

Model performance was compared and evaluated using leave-one-out cross validation. Leave-one-out validation consists of dividing the data into subsets by observation, and then training he models on data from all observations except for the one that is used for testing. Since each participant had two observation sessions, we also used leaveone-participant-out validation to verify that no bias exists towards participants.

\subsection{Evaluation Methods}

The objective of our work is to determine an individual's behavior on a second-bysecond basis. Because we are trying to make predictions at such a granular level, we measure our results in two ways.

\subsubsection{Micro-level Evaluation}

First, we measure our results on a micro-level. This is in terms of how well our models can predict a person's behavior on a second-by-second level. We evaluate this using classification accuracy precision, and recall. Classification accuracy for this work

is simply: $\frac{\text { the number of correctly predicted seconds }}{\text { the total number of observation seconds }}$. Precision is the percentage of true positives in the set of all positively classified samples, and recall is defined as the percentage of true positive in the set of all actual positive samples.

\subsubsection{Macro-level Evaluation}

We also measure our results on a macro-level. That is, we are interested in looking at the amount of time spent in each activity domain, or posture. We evaluate this 
by comparing the true proportions of the observed activities to our models' predicted proportions using a measure called Kullback-Leibler divergence [35]. Kullback-Leibler divergence, or simply called KL divergence, is a measure of the difference between two probability distributions $p(x)$ and $q(x)$. Let $p(x)$ be the actual distribution of classes and $q(x)$ be the predicted distribution. The KL divergence of $p(x)$ and $q(x)$ is defined as:

$$
D_{K L}(p(x) \| q(x))=\sum_{x \in X} p(x) \ln \frac{p(x)}{q(x)}
$$

\subsection{Model Results}

We test a range of models on each coding scheme and observe model performance as we increase the granularity of our class variables.

\subsubsection{Sedentary vs. Non-Sedentary Coding Results}

For our binary classification, we ran $K \mathrm{NN}$, SVM, random forest, and gradient boosting on our monitors. Table 5.1 reports the performance of our models on the Actigraph and BioStamp monitor data using the seven Staudenmayer features.

\begin{tabular}{|l|l|l|l|l|}
\hline \multirow{2}{*}{ Model } & \multicolumn{4}{|c|}{ Accuracy (\%) } \\
\cline { 2 - 5 } & $\begin{array}{l}\text { Actigraph } \\
\text { Wrist }\end{array}$ & $\begin{array}{l}\text { BioStamp Thigh } \\
\text { and Chest }\end{array}$ & $\begin{array}{l}\text { BioStamp } \\
\text { Thigh }\end{array}$ & $\begin{array}{l}\text { BioStamp } \\
\text { Chest }\end{array}$ \\
\hline \hline K-Nearest Neighbors & 63.72 & 98.61 & $\mathbf{9 8 . 6 2}$ & 76.38 \\
\hline SVM & 47.14 & 89.44 & 94.58 & 50.97 \\
\hline Random Forest & 76.42 & 98.54 & 98.51 & 77.17 \\
\hline Gradient Boosting & 75.59 & 98.13 & 98.07 & 76.04 \\
\hline
\end{tabular}

Table 5.1: Model performance predicting sedentary time using the 7 features from [56].

In terms of what monitor and classifier best predicts sedentary versus non-sedentary time - the best performance was demonstrated by the BioStamp thigh monitor and combined thigh and chest monitors across all classifiers, while the Actigraph wrist 


\begin{tabular}{|c|c|c|c|c|}
\hline & \multicolumn{4}{|c|}{ Predicted } \\
\hline \multirow{4}{*}{ Actual } & & Sedentary & Non-sedentary & Recall \\
\cline { 2 - 5 } & Sedentary & $\mathbf{1 3 5 4 9 1}$ & 40857 & 0.768 \\
\cline { 2 - 5 } & Non-sedentary & 41898 & $\mathbf{1 3 2 7 4 2}$ & 0.76 \\
\cline { 2 - 5 } & Precision & 0.764 & 0.765 & 0.764 \\
\hline
\end{tabular}

Table 5.2: Overall confusion matrix for RF predicting sedentary time from wrist Actigraph.

\begin{tabular}{|c|c|c|c|c|}
\hline & \multicolumn{4}{|c|}{ Predicted } \\
\hline \multirow{4}{*}{ Actual } & & Sedentary & Non-sedentary & Recall \\
\cline { 2 - 5 } & Sedentary & $\mathbf{1 7 8 6 5 4}$ & 2082 & 0.988 \\
\cline { 2 - 5 } & Non-sedentary & 3098 & $\mathbf{1 7 1 0 7 8}$ & 0.982 \\
\cline { 2 - 5 } & Precision & 0.983 & 0.988 & 0.985 \\
\hline
\end{tabular}

Table 5.3: Overall confusion matrix for RF predicting sedentary time from thigh and chest BioStamps.

monitor gave us the worst performance. Tables 5.2 and 5.3 show the confusion matrices for the wrist Actigraph and the thigh and chest BioStamps. The wrist's lower accuracy can be due to the amount of noise the wrist monitor records, as wrist movement does not necessarily directly correspond to an individual's behavior. In addition, the chest BioStamp on its own performed similarly to the wrist on most classifiers, with the exception of $K \mathrm{NN}$ as the chest performed better. The lower accuracy of the chest monitor can be attributed to the fact that some sedentary behaviors look very similar to non-sedentary behaviors with respect to the individual's chest (i.e., sitting versus standing).

We also evaluated model accuracy on a larger scale with respect to activity domains. Figure 5.1 shows a comparison of our random forest classifier's predictions of sedentary time on the Actigraph wrist monitor and the combined thigh and chest BioStamp monitors. Color-coded by activity domain, each point represents one twohour observation session. The random forest using the thigh and chest monitors has an almost perfect direct relationship, with a regression coefficient of 0.9992 . The wrist-fitted random forest performs reasonably well, with a regression coefficient of 


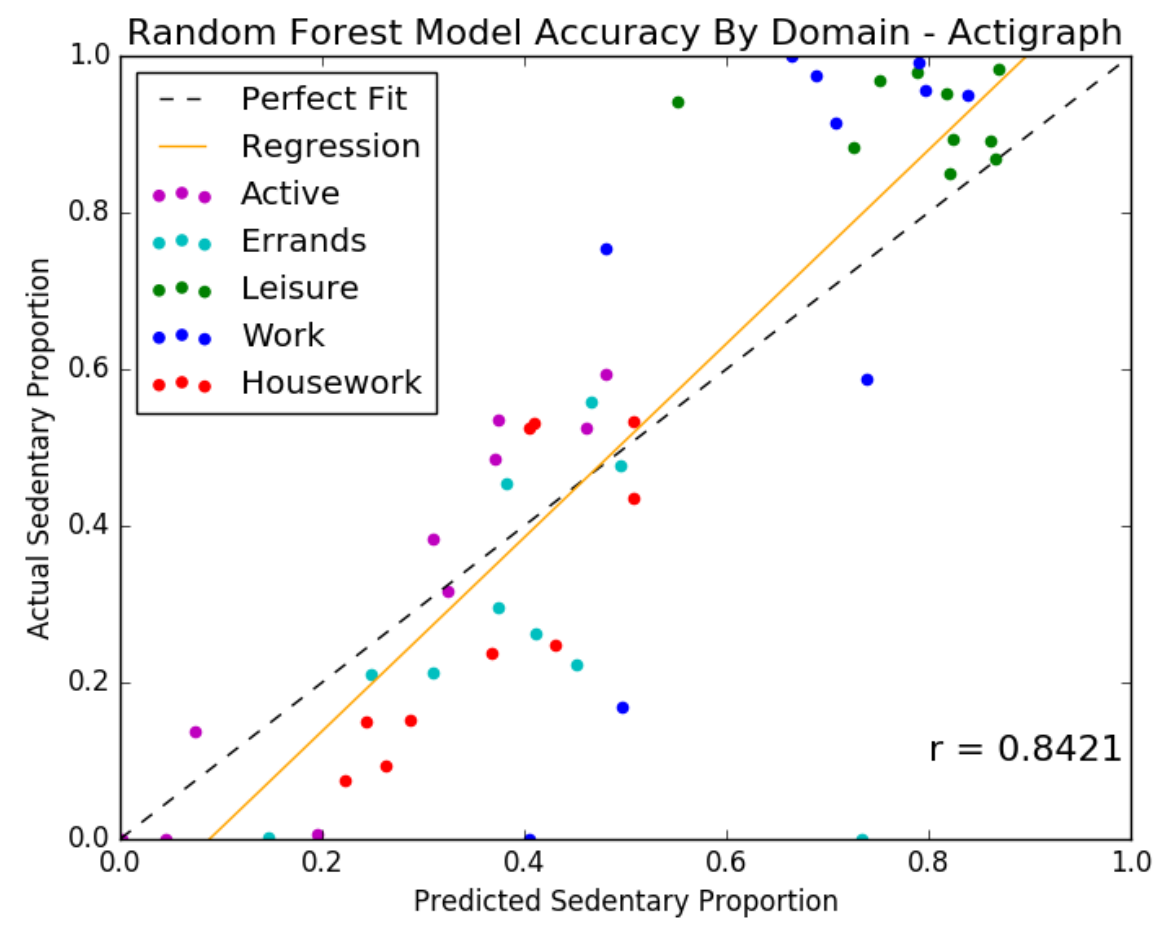

(a) Actigraph wrist

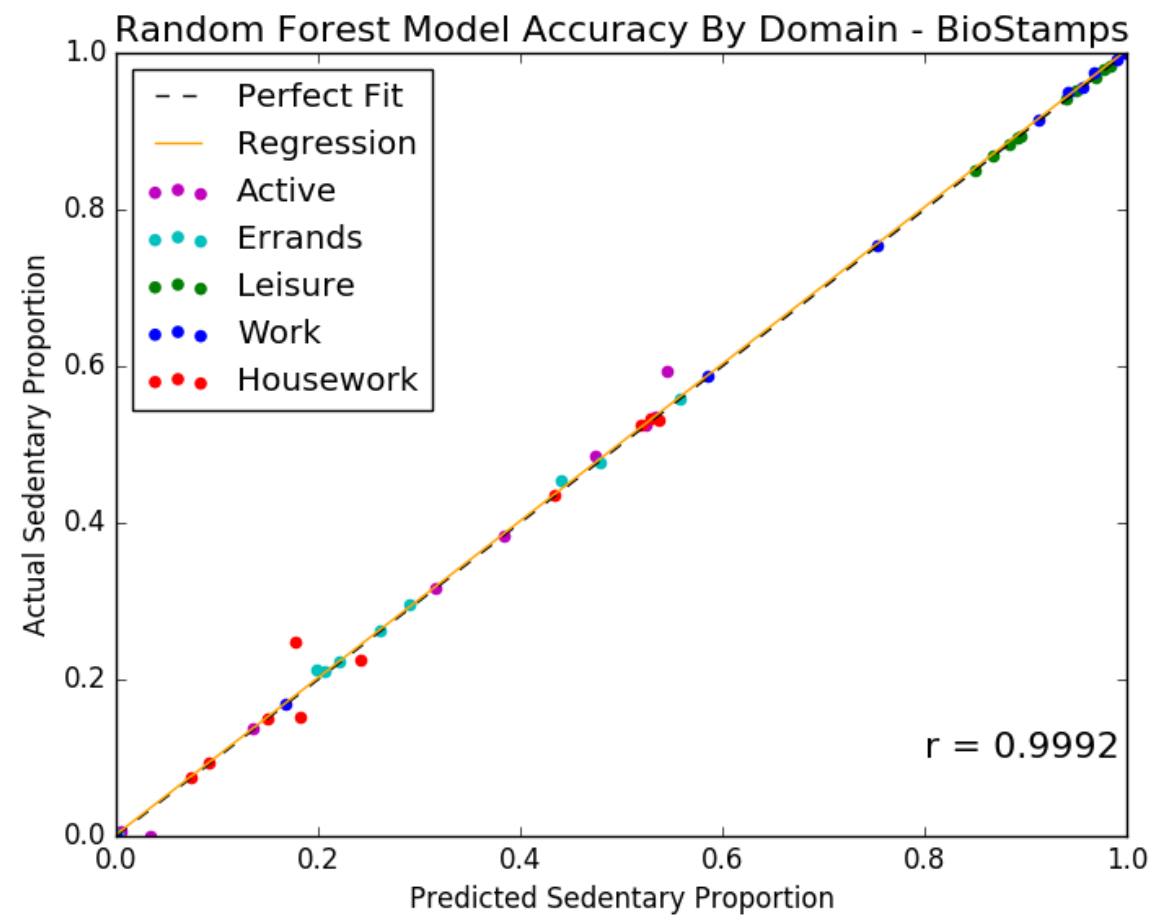

(b) BioStamp thigh and chest

Figure 5.1: Actual by predicted sedentary time by activity domain. 
0.8421.

To see how our models compare, we ran the random forest model from Staudenmayer et. al [56] on our wrist Actigraph data. The Staudenmayer method predicted sedentary time with $70.86 \%$ accuracy. Our random forest and gradient boosting models achieve higher accuracies than the Staudenmayer method on our wrist Actigraph data, which further supports the claim that models trained on free-living data outperform lab-trained models.

Remember that we created 16 additional features to attempt to provide more information about the accelerometer signals to our models. We found that adding more features improved sedentary/non-sedentary classification accuracy by up to $6.3 \%$, in the case of the combined thigh and chest BioStamps' SVM (shown in Appendix C). To make sure our models were achieving the best possible accuracy scores, the total 23 features were used for the reported accuracies of all other coding schemes.

\subsubsection{Activity Intensity (METs) Results}

Seeing as prior work $[61,56]$ has predicted energy expenditure from accelerometer data and metabolic equivalents (METs) are known to be the most common unit for measuring activity intensity, we aggregated the predictions of our most granular, 14class coding scheme in accordance with Table 5.4. The aggregated results of our models for the activity intensity coding scheme are shown in Table 5.5. We observe that the models we trained to learn 14 posture classes, can accurately recognize ac-

\begin{tabular}{|l|l|}
\hline Intensity Level & \# Postures \\
\hline \hline Sedentary & Lying down, sitting, kneeling/squatting \\
\hline Light & Stand, stand and move \\
\hline Moderate & Walk, ascending stairs, descending stairs \\
\hline Vigorous & Sports, running, biking \\
\hline
\end{tabular}

Table 5.4: Postures that fall under each intensity label. 


\begin{tabular}{|l|l|l|l|l|}
\hline \multirow{2}{*}{ Model } & \multicolumn{3}{|c|}{ Accuracy (\%) } \\
\cline { 2 - 5 } & $\begin{array}{l}\text { Actigraph } \\
\text { Wrist }\end{array}$ & $\begin{array}{l}\text { BioStamp Thigh } \\
\text { and Chest }\end{array}$ & $\begin{array}{l}\text { BioStamp } \\
\text { Thigh }\end{array}$ & $\begin{array}{l}\text { BioStamp } \\
\text { Chest }\end{array}$ \\
\hline \hline K-Nearest Neighbors & 53.43 & 94.07 & 93.93 & 93.61 \\
\hline SVM & 39.33 & 89.8 & 90.91 & 84.96 \\
\hline Random Forest & 68.08 & $\mathbf{9 4 . 6 7}$ & 94.48 & 94.33 \\
\hline
\end{tabular}

Table 5.5: Model performance predicting activity intensity (METs) from aggregating our 14-class models.

tivity intensity. In terms of what monitor and ML method best predicts activity intensity, all BioStamp monitors performed comparably to one another on all classifiers, while the wrist Actigraph still gave us a significantly lower accuracy. We also observe that the chest BioStamp was able to more accurately estimate activity intensity than sedentary time, which demonstrates that more vigorous activities can be distinguished from more sedentary activities from chest movement.

We also trained some new models to learn activity intensity. Although the research team had already coded intensity with respect to each posture in the full coding scheme, we defined intensities by posture type for these models consistently with our aggregated results (Table 5.4). As shown in Table 5.6, re-training new models provides similar classification accuracies to the aggregated results of our full coding scheme models.

We used KL divergence to evaluate our models with respect to the true proportion

\begin{tabular}{|l|l|l|l|l|}
\hline \multirow{2}{*}{ Model } & \multicolumn{4}{|c|}{ Accuracy (\%) } \\
\cline { 2 - 5 } & $\begin{array}{l}\text { Actigraph } \\
\text { Wrist }\end{array}$ & $\begin{array}{l}\text { BioStamp Thigh } \\
\text { and Chest }\end{array}$ & $\begin{array}{l}\text { BioStamp } \\
\text { Thigh }\end{array}$ & $\begin{array}{l}\text { BioStamp } \\
\text { Chest }\end{array}$ \\
\hline \hline KNN aggregated & 53.43 & 94.07 & 93.93 & 93.61 \\
\hline KNN retrained & 53.19 & 94.07 & 93.99 & 93.63 \\
\hline RF aggregated & 68.08 & 94.67 & 94.48 & 94.33 \\
\hline RF retrained & 69.08 & 94.47 & 94.55 & 94.18 \\
\hline
\end{tabular}

Table 5.6: Classification accuracies predicting activity intensities (METs) from aggregating our 14-class models vs. our retrained models. 
of activity intensity levels versus our predicted proportion. Figure 5.2 shows the actual and predicted probability distributions for our activity intensity coding scheme, and Table 5.7 compares our aggregated 14-class models with our retrained models in terms of KL divergence. Recall the KL divergence is 0 if our predicted class distribution is the exact same as the actual class distribution of data. The KL divergence between our aggregated vs. retrained models show similar distributions. This is a significant discovery; the aggregation method lets us train one model to learn a more granular set of class variables and can still achieve competitive results on less granular coding schemes to models that have been retrained on the less granular coding scheme. Sometimes the aggregation method even provides better results than the retrained models.

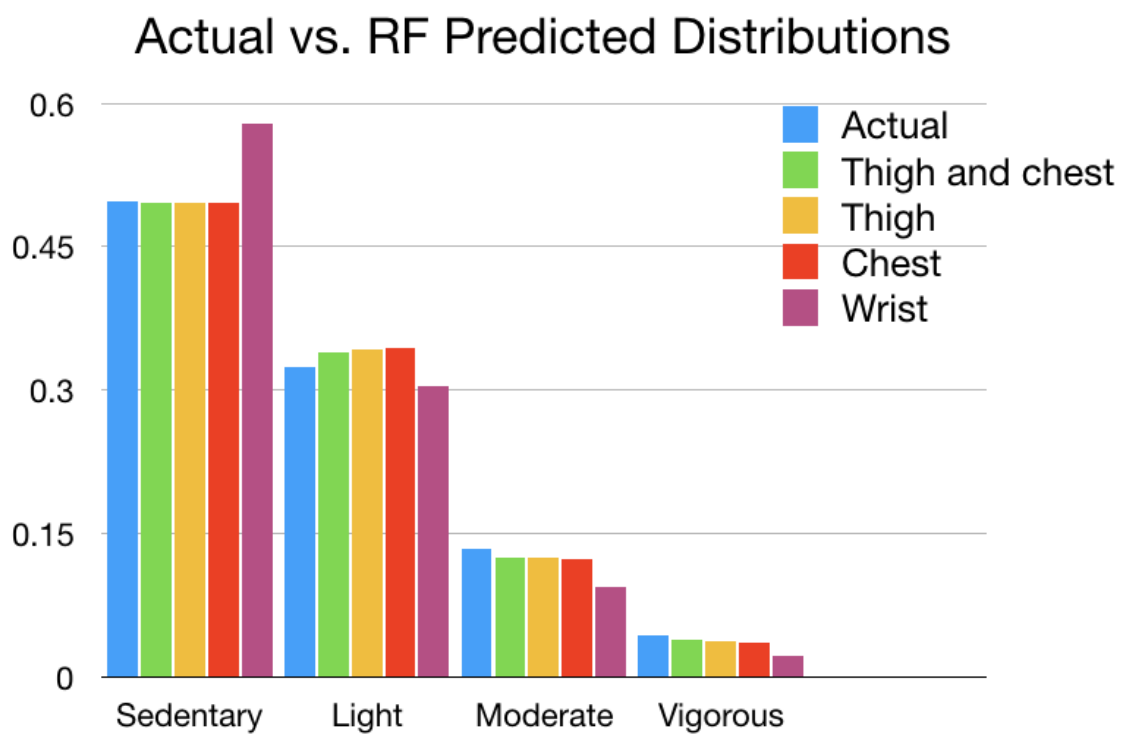

Figure 5.2: Actual vs. predicted probability distributions of random forests predicting activity intensity. 


\begin{tabular}{|l|l|l|l|l|}
\hline \multirow{2}{*}{ Model } & \multicolumn{4}{|c|}{ Accuracy (\%) } \\
\cline { 2 - 5 } & $\begin{array}{l}\text { Actigraph } \\
\text { Wrist }\end{array}$ & $\begin{array}{l}\text { BioStamp Thigh } \\
\text { and Chest }\end{array}$ & $\begin{array}{l}\text { BioStamp } \\
\text { Thigh }\end{array}$ & $\begin{array}{l}\text { BioStamp } \\
\text { Chest }\end{array}$ \\
\hline \hline KNN aggregated & 0.0761 & 0.0002 & 0.00008 & 0.0002 \\
\hline KNN retrained & 0.0029 & 0.0002 & 0.0001 & 0.0002 \\
\hline RF aggregated & 0.0191 & 0.001 & 0.0014 & 0.0016 \\
\hline RF retrained & 0.018 & 0.0013 & 0.001 & 0.0019 \\
\hline
\end{tabular}

Table 5.7: KL divergence of our aggregated 14-class models vs. our retrained models.

\subsubsection{General Postures Results}

Since our full coding scheme is significantly more detailed than those used in previous work, we generalized postures down to a total of five posture labels in order to more directly compare our results with other work. Ellis et. al [20] classified free-living wrist accelerometer data into four activity classes: sit, stand, walk/run, and riding in a vehicle; we used these activity classes as four out of five of our postures in this general coding scheme. Our dataset included observations that did not fall into any of these classes, so we added an other class. The postures from the full coding scheme are absorbed into these five activity classes as described in Table 5.8. The classification accuracies of our retrained models on this general postures coding scheme is reported in Table 5.9. Aggregating results from our 14-posture class models proved to be unfeasible, simply because riding in a vehicle in our original ground truth is coded as a traveling behavior, not a posture. This information was dropped while training

\begin{tabular}{|l|l|}
\hline General Posture Coding & Description/Postures from Full Coding Scheme \\
\hline \hline Sit & Lying down, sitting, kneeling/squatting \\
\hline Stand & Stand, stand and move \\
\hline Walk/Run & Walk, ascending stairs, descending stairs, running \\
\hline Riding in Vehicle & $\begin{array}{l}\text { Traveling behaviors - driving or riding in a } \\
\text { car/truck/motorcycle }\end{array}$ \\
\hline Other & Sports, biking \\
\hline
\end{tabular}

Table 5.8: Postures/behaviors that are considered for each general posture. 


\begin{tabular}{|l|l|l|l|l|}
\hline \multirow{2}{*}{ Model } & \multicolumn{4}{|c|}{ Accuracy (\%) } \\
\cline { 2 - 5 } & $\begin{array}{l}\text { Actigraph } \\
\text { Wrist }\end{array}$ & $\begin{array}{l}\text { BioStamp Thigh } \\
\text { and Chest }\end{array}$ & $\begin{array}{l}\text { BioStamp } \\
\text { Thigh }\end{array}$ & $\begin{array}{l}\text { BioStamp } \\
\text { Chest }\end{array}$ \\
\hline \hline K-Nearest Neighbors & 53.57 & 93.6 & 93.49 & 93.18 \\
\hline SVM & 49.55 & 80.66 & 79.59 & 76.22 \\
\hline Random Forest & 68.92 & $\mathbf{9 3 . 8 1}$ & 94.08 & 93.55 \\
\hline
\end{tabular}

Table 5.9: Model performance predicting the general coding scheme with 5 posture labels.

our most granular 14-class models, and therefore is no longer available to aggregate by.

Similar to our results for the activity intensity coding scheme, all of the BioStamp models outperform the Actigraph models. Given the classification accuracies that our models achieved predicting our five activity classes, we conclude that our methods outperform Ellis et al., as they obtained a balanced accuracies of $89.4 \%$ and $84.6 \%$ from their hip and wrist accelerometers for predicting four activity classes. Recall that Ellis et al. used one-minute interval windows, while our models use intervals of one second. One minute intervals would most likely further increase our model performance. It is also worth noting that our ground truth was constructed through a very thorough and detailed coding process using a GoPro Hero 5 video camera; a SenseCam that captured still images approximately every 20 seconds served as the ground truth for [20]. Therefore it is likely that the ground truth labels in [20] are not entirely accurate in a handful of cases. For instance, a participant could easily transition from sitting in a chair, to walking across a room, back to sitting in a chair within a 20-second time span. The SenseCam would have, and likely did, miss capturing these behaviors.

Tables 5.10 and 5.11 report the confusion matrices of our best and worst models. We observe that the thigh and chest BioStamps' random forest classifier is able to recognize the other postures much better than the wrist's $K \mathrm{NN}$ classifier. We also 


\begin{tabular}{|l|l|l|l|l|l|l|l|}
\hline & \multicolumn{7}{|c|}{ Predicted } \\
\hline & & sit & standing & walk/run & $\begin{array}{l}\text { riding in } \\
\text { vehicle }\end{array}$ & other & Recall \\
\cline { 2 - 9 } & sit & $\mathbf{1 6 7 8 3 6}$ & 2286 & 293 & 87 & 25 & 0.9842 \\
\cline { 2 - 9 } Actual & standing & 1489 & $\mathbf{1 0 6 4 3 4}$ & 4302 & 0 & 50 & 0.948 \\
\cline { 2 - 9 } & walk/run & 326 & 9156 & $\mathbf{4 6 1 7 6}$ & 0 & 36 & 0.829 \\
\cline { 2 - 9 } & $\begin{array}{l}\text { riding in } \\
\text { vehicle }\end{array}$ & 1460 & 15 & 45 & $\mathbf{0}$ & 0 & 0 \\
\cline { 2 - 9 } & other & 354 & 891 & 608 & 0 & $\mathbf{4 1 9 1}$ & 0.6934 \\
\cline { 2 - 9 } & Precision & 0.9788 & 0.896 & 0.8979 & 0 & 0.9742 & \\
\hline
\end{tabular}

Table 5.10: Confusion matrix for RF predicting 5 general activity classes using the thigh and chest BioStamps.

\begin{tabular}{|l|l|l|l|l|l|l|l|}
\hline & \multicolumn{9}{|c|}{ Predicted } \\
\hline \multirow{5}{*}{ Actual } & sit & standing & walk/run & $\begin{array}{l}\text { riding in } \\
\text { vehicle }\end{array}$ & other & 0.7872 \\
\cline { 2 - 9 } & sit & $\mathbf{1 1 3 5 6 4}$ & 46066 & 9744 & 594 & 1019 & 0.6068 \\
\cline { 2 - 9 } & standing & 52031 & $\mathbf{4 2 0 4 2}$ & 12711 & 171 & 1056 & \\
\cline { 2 - 9 } & walk/run & 14390 & 15992 & $\mathbf{2 3 1 0 7}$ & 24 & 2099 & 0.6413 \\
\cline { 2 - 9 } & $\begin{array}{l}\text { riding in } \\
\text { vehicle }\end{array}$ & 1073 & 401 & 41 & $\mathbf{0}$ & 5 & 0 \\
\cline { 2 - 9 } & other & 1736 & 1908 & 2397 & 3 & $\mathbf{0}$ & 0 \\
\cline { 2 - 9 } & Precision & 0.756 & 0.5331 & 0.5331 & 0 & 0 & \\
\hline
\end{tabular}

Table 5.11: Confusion matrix for $K N N$ classifier predicting 5 general activity classes using wrist Actigraph.

find that neither of these models capture riding in a vehicle at all. However, both models predicted sit the most for these observations. Given that a person is typically sitting while riding in a vehicle, we consider this misclassification acceptable.

\subsubsection{Full Coding Scheme Results}

Dr. Keadle was also interested in increasing the granularity of our class variables and seeing whether our models could accurately recognize 14 posture classes. The accuracy of our models on the full coding scheme of fourteen class labels is presented in Table 5.12. We see a significant drop in performance as we increase the granularity of our class variables. However, our models continue to perform consistently with respect 


\begin{tabular}{|l|l|l|l|l|}
\hline \multirow{2}{*}{ Model } & \multicolumn{3}{|c|}{ Accuracy (\%) } \\
\cline { 2 - 5 } & $\begin{array}{l}\text { Actigraph } \\
\text { Wrist }\end{array}$ & $\begin{array}{l}\text { BioStamp Thigh } \\
\text { and Chest }\end{array}$ & $\begin{array}{l}\text { BioStamp } \\
\text { Thigh }\end{array}$ & $\begin{array}{l}\text { BioStamp } \\
\text { Chest }\end{array}$ \\
\hline \hline K-Nearest Neighbors & 50.49 & 85.37 & 85.38 & 85.02 \\
\hline SVM & 43.94 & 78.87 & 79.87 & 80.37 \\
\hline Random Forest & 61.61 & $\mathbf{8 5 . 9 8}$ & 85.73 & 85.47 \\
\hline
\end{tabular}

Table 5.12: Model performance on the full coding scheme.

how they compare with one another. Our wrist models are again outperformed by all of the BioStamps. In this specific case, the best predictor of posture is the random forest using the combined thigh and chest data.

The confusion matrix for our combined thigh and chest random forest is in Table 5.13. Here we can closely observe the class imbalance problem within our dataset. Firstly, our models have almost no chance of learning lying or other sport postures, given that lying only has 25 observation seconds and other sport only has 2. On the other end of the spectrum, sitting has the largest observation sample size with 171985 seconds. Our random forest also seemed to favor predicting stand and move light more than the other intensities stand and move moderate and stand and move vigorous. This can be attributed to the larger sample size of the stand and move light class. The class imbalance problem is also exhibited through the different intensities of walking; walk moderate got the majority of our model's predictions as it has a larger sample size than the other intensities. Table 5.14 presents the precision, recall, and F-measures (harmonic mean of precision and recall) for each posture class along with the number of samples and their percentage of our dataset; the classes are plotted according to their percentage of the dataset and their F-measures on a log scale in Figure 5.3. We observe that the more samples a class has in the dataset, the better the F-measure.

Reviewing our results at a macro-level, we calculated the KL divergence of our random forest models on each posture class and present them in Figure 5.4. KL 


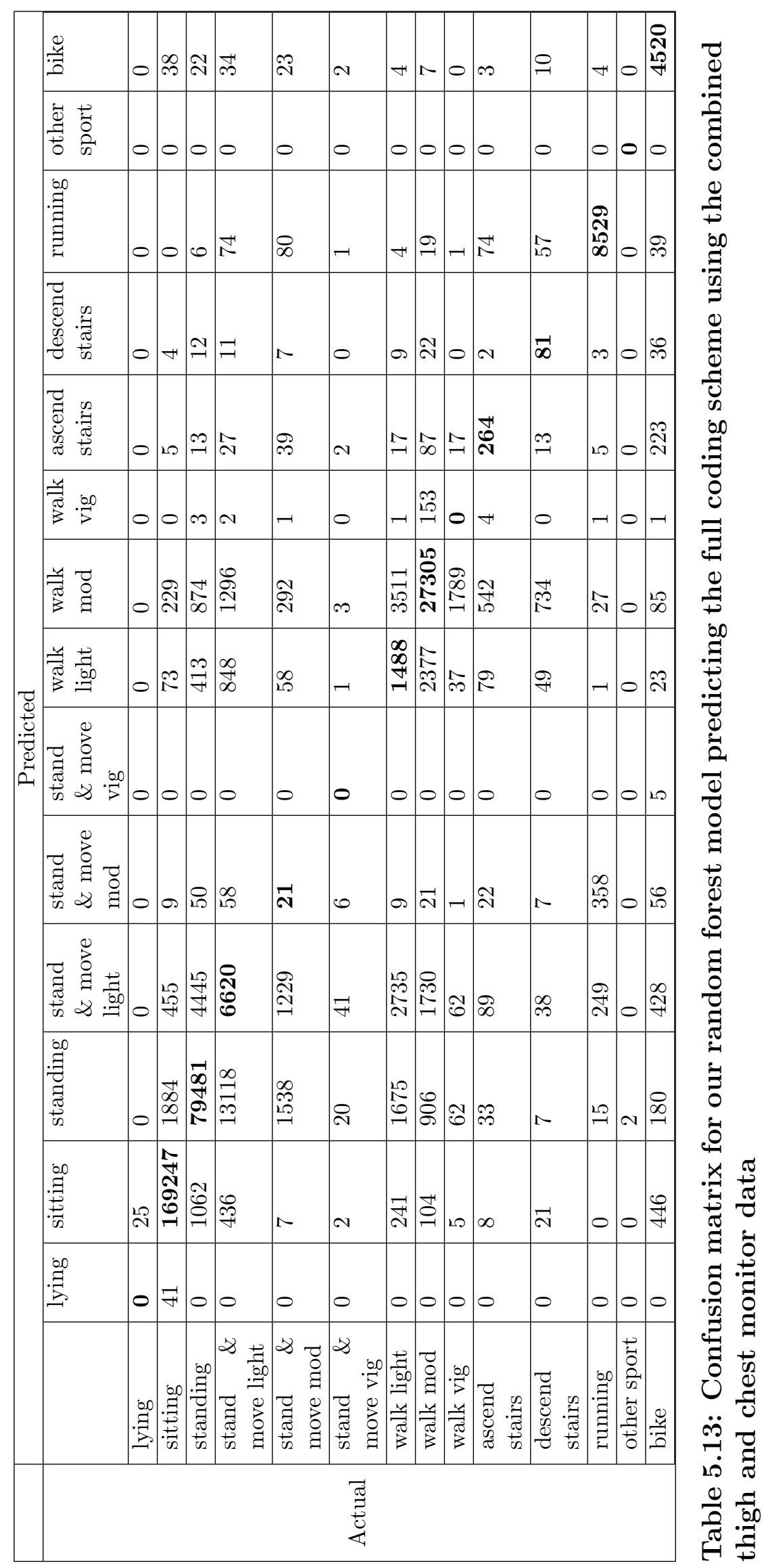




\begin{tabular}{|l|c|c|c|c|c|}
\hline Posture class & Precision & Recall & F-measure & \# samples & \% of dataset \\
\hline \hline Sitting & 0.9862 & 0.9841 & 0.9852 & 171985 & 0.49698 \\
\hline Standing & 0.8035 & 0.9201 & 0.8579 & 86381 & 0.249613 \\
\hline Walk moderate & 0.7443 & 0.8342 & 0.7867 & 32731 & 0.094582 \\
\hline Stand and move light & 0.3653 & 0.2939 & 0.3257 & 22524 & 0.065087 \\
\hline Walk light & 0.2732 & 0.1535 & 0.1966 & 9694 & 0.028012 \\
\hline Running & 0.96 & 0.9279 & 0.9437 & 9192 & 0.026562 \\
\hline Bike & 0.9685 & 0.7481 & 0.8441 & 6042 & 0.017459 \\
\hline $\begin{array}{l}\text { Stand and move mod- } \\
\text { erate }\end{array}$ & 0.034 & 0.0064 & 0.0107 & 3295 & 0.009521 \\
\hline Walk vigorous & 0 & 0 & 0 & 1974 & 0.005704 \\
\hline Ascending stairs & 0.3708 & 0.2357 & 0.2882 & 1120 & 0.003236 \\
\hline Descending stairs & 0.433 & 0.0796 & 0.1346 & 1017 & 0.002939 \\
\hline $\begin{array}{l}\text { Stand and move vigor- } \\
\text { ous }\end{array}$ & 0 & 0 & 0 & 78 & 0.000225 \\
\hline Lying & 0 & 0 & 0 & 25 & 0.000072 \\
\hline Sports & 0 & 0 & 0 & 2 & 0.000006 \\
\hline
\end{tabular}

Table 5.14: Precision, recall, and f-measures of our thigh and chest BioStamps random forest, sorted by class proportion to the dataset.

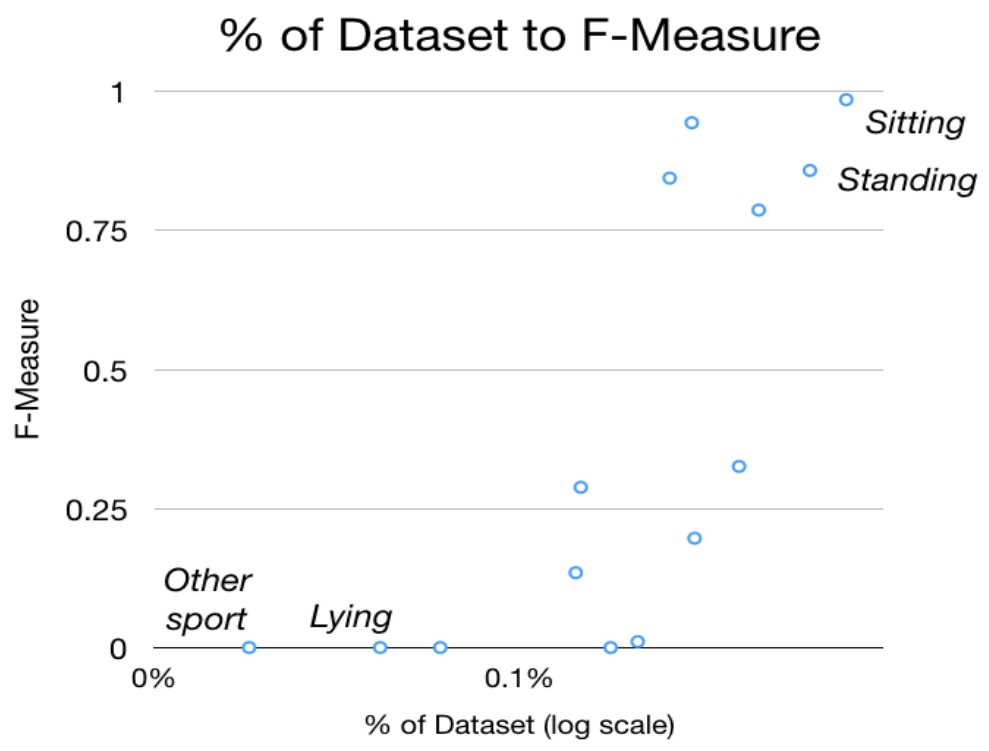

Figure 5.3: Posture proportions to our dataset by their F-measures. 


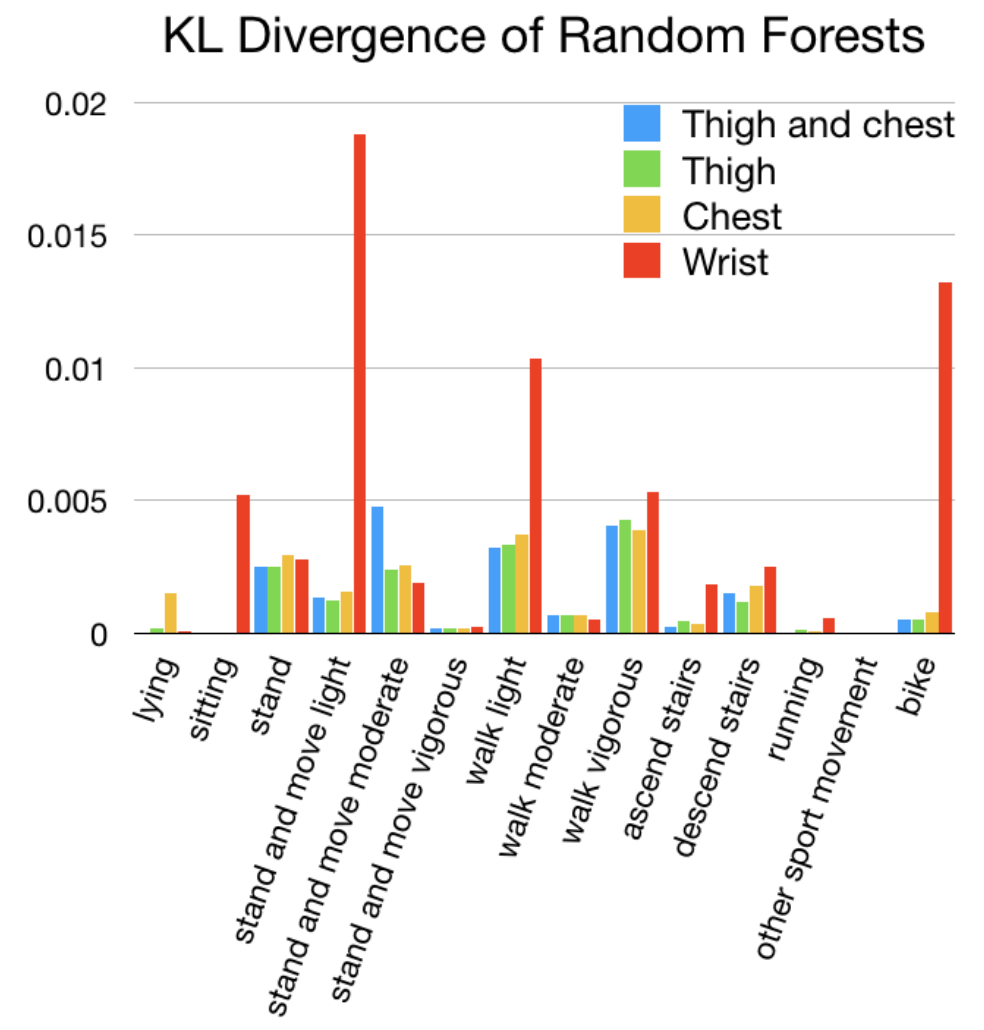

Figure 5.4: KL divergence of our random forest models.

divergence is a measure of how different two probability distributions are - the closer to 0 , the more similar our models are to our ground truth. This graph also allows us to notice the differences in our model predictions using different monitors, specifically the difference between our wrist method and the BioStamps.

Another one of our research questions asked whether using a hierarchical classification schema would improve performance. We were specifically interested in finding out whether the presence of other data made the differences between certain postures less recognizable. Therefore, we attempted to improve classification accuracy by structuring our posture classes in a hierarchy and training classifiers to distinguish between more specific postures. Table 5.15 shows a comparison of the differences in classification accuracy from our flat random forest classifiers to our hierarchical 
approach using random forests. As shown, the hierarchy did not provide any lift in classification accuracy.

\begin{tabular}{|l|l|l|l|l|}
\hline \multirow{2}{*}{ Model } & \multicolumn{3}{|c|}{ Accuracy (\%) } \\
\cline { 2 - 5 } & $\begin{array}{l}\text { Actigraph } \\
\text { Wrist }\end{array}$ & $\begin{array}{l}\text { BioStamp Thigh } \\
\text { and Chest }\end{array}$ & $\begin{array}{l}\text { BioStamp } \\
\text { Thigh }\end{array}$ & $\begin{array}{l}\text { BioStamp } \\
\text { Chest }\end{array}$ \\
\hline \hline Random Forest & 61.61 & 85.98 & 85.73 & 85.47 \\
\hline $\begin{array}{l}\text { Random Forest } \\
\text { Hierarchy }\end{array}$ & 61.21 & 85.11 & 85.15 & 84.88 \\
\hline
\end{tabular}

Table 5.15: Flat random forest classifier versus random forest ensemble hierarchy performance.

One main disadvantage of our hierarchical classification approach is that misclassifications made at higher levels of the hierarchy are inherited by the classifiers at the lower levels. For instance, the sitting observation samples that the root classifier classified as stand, will not be corrected by the standing classifier since it only predicts between stand and stand and move. Table 5.16 shows the confusion matrix for our random forest classifier predicting the postures in the first class level of the posture hierarchy using the combination of the thigh and chest BioStamps; the final confusion matrix of our hierarchical random forest ensemble predicting all 14 postures is in Table 5.17 .

In addition to the standard accuracy measure of the percentage of correctly predicted observations, we also evaluated model performance using the mean absolute error metric (MAE) and the macro-averaged mean absolute error metric (MA-MAE). MAE is a commonly used loss function that equals the sum of the absolute differences between our ground truth and predicted variables. Given a vector $\mathbf{v}=\left(v_{1}, \ldots, v_{n}\right)$ of ground truth values and a vector of predictions $\mathbf{p}=\left(p_{1}, \ldots, p_{n}\right)$, the prediction's MAE is calculated as

$$
M A E(p, v)=\frac{1}{n} \sum_{i=1}^{n}\left|p_{i}-v_{i}\right|
$$

In other words, the MAE of a prediction represents the average magnitude of errors 


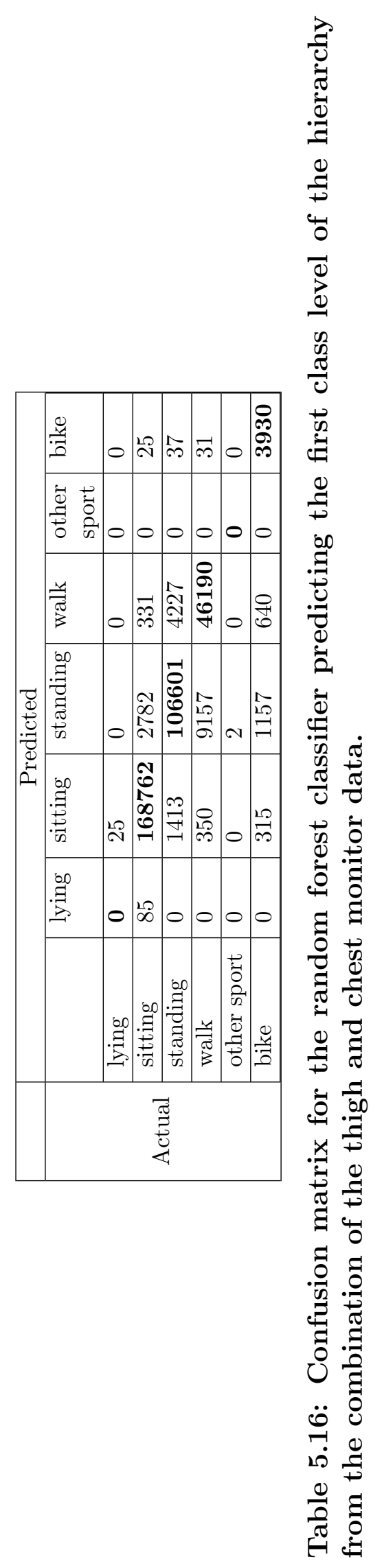




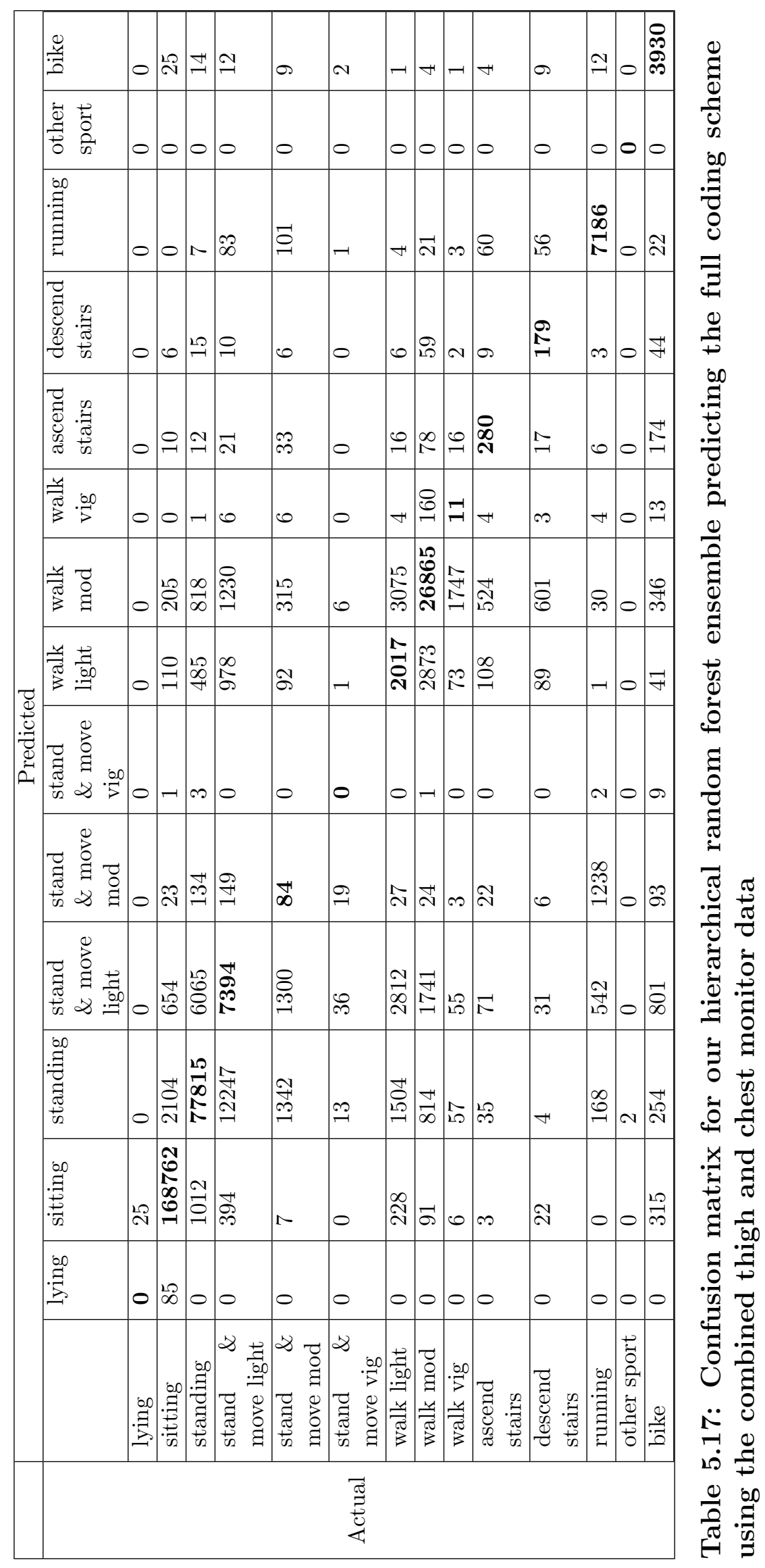


from a set of predictions, regardless of the direction of error. MAE can range from 0 to $\infty$, with the best value being 0 . Because MAE has no maximum bound, we also use a variant of the MAE metric that scores between $[0,1]$, called the macro-averaged mean absolute error (MA-MAE). This metric is used on ordinal classes and aims to punish certain errors more based on the severity of the error. For instance, in terms of our classification problem, misclassifying lying as running would be considered a severe error and would be penalized more by MA-MAE, while misclassifying stand and move light for stand and move moderate would be a less severe error and penalized less. The MA-MAE for this experiment is computed by separating the test set into 14 subsets - one per posture class - then independently calculating each class category's MAE, and finally combining the normalized MAEs into a weighted sum, which is subtracted from 1 to be consistent with the "higher is better" intuition. The MAEs of each subset are normalized by the highest possible error for a data point with the given posture. Since MA-MAE is a metric used on ordinal classes, we made our ground truth labels ordinal as defined in Table 5.18.

\begin{tabular}{|l|l|}
\hline Posture Coding & Ordinal Coding \\
\hline \hline Lying down & 0 \\
\hline Sitting & 1 \\
\hline Standing & 2 \\
\hline Stand and move light & 3 \\
\hline Stand and move moderate & 4 \\
\hline Stand and move vigorous & 5 \\
\hline Walk light & 6 \\
\hline Walk moderate & 7 \\
\hline Walk vigorous & 8 \\
\hline Ascending stairs & 9 \\
\hline Descending stairs & 10 \\
\hline Running & 11 \\
\hline Sports & 12 \\
\hline Bike & 13 \\
\hline
\end{tabular}

Table 5.18: The ordinal coding of the posture classes.

With this ordinal coding of postures, the highest possible error would be from 
misclassifying lying down as sports, or vice versa. The normalization factor in these misclassification cases is $|0-13|=13$, or $|13-0|=13$. As another example, the highest possible error for walk moderate is $|7-0|=7$. Our MA-MAE formula is therefore:

$M A \_M A E(p, v)=1-\frac{\left.\left(\frac{M A E\left(p_{0}, v_{0}\right)}{13}+\ldots+\frac{M A E\left(p_{6}, v_{6}\right)}{7}+\frac{M A E\left(p_{7}, v_{7}\right)}{7}+\ldots+\frac{M A E\left(p_{13}, v_{13}\right.}{13}\right)\right)}{14}$

We compare the MA-MAEs for our random forest and hierarchical random forest classifiers. In this case, our hierarchical approach achieved a better MA-MAE score for the wrist and the thigh models, and fell slightly short with the chest and the thigh and chest models. By changing the nature of error, we see that although our hierarchical classifier predicted more errors in all cases, it predicts less severe errors in some cases.

\begin{tabular}{|l|l|l|l|l|}
\hline \multirow{2}{*}{ Model } & \multicolumn{4}{|c|}{ MA-MAE } \\
\cline { 2 - 5 } & $\begin{array}{l}\text { Actigraph } \\
\text { Wrist }\end{array}$ & $\begin{array}{l}\text { BioStamp Thigh } \\
\text { and Chest }\end{array}$ & $\begin{array}{l}\text { BioStamp } \\
\text { Thigh }\end{array}$ & $\begin{array}{l}\text { BioStamp } \\
\text { Chest }\end{array}$ \\
\hline \hline Random Forest & 0.6697 & 0.8051 & 0.8013 & 0.7943 \\
\hline $\begin{array}{l}\text { Random Forest } \\
\text { Hierarchy }\end{array}$ & 0.6854 & 0.8007 & 0.8033 & 0.7934 \\
\hline
\end{tabular}

Table 5.19: MA-MAE comparison of models.

Using AdaBoost also failed to provide us with the lift we hoped for. It is possible that because of the imbalanced nature of our dataset, even Adaboost struggled to learn certain classes that had a significantly smaller sample size. The confusion matrix for AdaBoost with the thigh and chest BioStamp is shown in Table B.15, and can be found in Appendix B.

When working with imbalanced classes, the classification accuracy may not be the ideal metric for us to optimize. As the confusion matrices demonstrate, models tend to favor the majority classes, while failing to recognize the minority classes. 
Our final investigation was to implement a multi-class boosting method that uses the confusion matrix as the optimizing measure and to observe whether classification accuracy increases. Our implementation of CoMBo combats the class imbalance problem by considering the number of samples for each class while updating the misclassification costs for each class, in addition to the level of difficulty associated with classifying a sample. Due to the time complexity that came with updating the score functions and cost matrices in this algorithm, we trained five CoMBo models one per activity domain - which allowed us to subset our data. A smaller training set made the cost matrices significantly smaller and therefore decreased training time. Although training time was decreased substantially, our models have not predicted every observation session by the end of this work. These preliminary results present accuracies missing about ten total observation sessions. Regardless of these several missing predictions, we believe our results are still informative. Table 5.20 shows the preliminary accuracies for each of the activity domains using leave-one-out cross validation.

\begin{tabular}{|l|l|}
\hline Activity Domain & Accuracy (\%) \\
\hline \hline Active & 80.74 \\
\hline Errands & 74.12 \\
\hline Leisure & 94.7 \\
\hline Household & 89.88 \\
\hline Work & 93.49 \\
\hline
\end{tabular}

Table 5.20: Preliminary CoMBo accuracies by activity domain for combined thigh and chest BioStamps. 
Chapter 6

\section{DISCUSSION AND CONCLUSION}

In this work, we developed models to predict a person's physical activity/posture based on three sets of monitor data. We ran experiments to answer questions posed by Dr. Keadle from Cal Poly's Department of Kinesiology and Public Health.

Question 1. Our first round of experiments was dedicated to finding out the best monitor placement and machine learning method for estimating sedentary versus non-sedentary behavior. In these experiments, we were able to achieve nearly perfect performance in predicting sedentary time within a given second, with the thigh $K \mathrm{NN}$ model achieving the highest accuracy (98.62\%) amongst our models in this particular case. In addition, we demonstrate that our models can almost perfectly recognize sedentary vs. non-sedentary behavior, no matter the activity domain.

Question 2. Another contribution of this thesis was to compare our methods to previously developed methods. We tested our dataset on Staudenmayer et al's random forest method, and found that our wrist model outperforms theirs. With this result, we are able to provide further support to the claim that laboratory-trained models are not the best predictors of free-living data.

Question 3. Dr. Keadle's third question required us to validate different monitors and monitor placements using different classifiers to estimate activity intensity. We observed two sets of results for this question. Our first set of results is referred to as our aggregated results. We took our most granular, 14-posture models and aggregated the predicted results from the 14-posture coding scheme into activity intensities. The 
other set of results is obtained from retraining new models for this activity intensity coding scheme. From observing two sets of results, we found that our most granular models estimate activity intensity equally as well as our models retrained the predict intensity levels. This is a significant contribution of our work as the aggregation method is more cost-effective, allowing us to only train one model that predicts a much granular coding scheme, while still accurately predicting a less granular coding scheme via aggregation. Taking both sets of results into consideration, the best predictor of activity intensity is our random forest classifier using the combined thigh and chest monitors.

Question 4. We were also interested in building predictive models to learn behaviors that were more specific than simply sedentary vs. non-sedentary. We created a new coding scheme to train on from our 14 class variables generalizing down to five activity classes, and obtained promising results in comparison to previous work [20]. In terms of what monitor and method best predicts the five general posture classes, our thigh and chest random forest model was the top contender for our experiments. However, all of our models failed to capture riding in a vehicle. This is due to the nonuniformity of our dataset, which is result of the nature of free-living data - humans tend to perform some activities more than others. Another reason riding in a vehicle was so difficult for our models to learn is because riding in a vehicle is essentially sitting.

Question 5. Dr. Keadle wanted to develop methods to predict activity type at an even more granular level than five postures. We developed models which correctly classified a participant's posture into 14 classes with up to $85.98 \%$ accuracy. In our particular set of experiments, we found that the combination of thigh and chest acceleration is the best indicator of physical posture and that random forest performs 
the best out of our traditional classifiers.

Question 6. Given that our 14 activity classes could be structured into a 3-level hierarchy, we used the local classifier per parent node approach and built a hierarchical ensemble of random forest classifiers. Although our attempt to use a hierarchical ensemble did not prove to be as successful as we had hoped - we found that changing our error measure to evaluate the severity of our misclassifications revealed that using a hierarchical classification approach could result in less severe errors than some of our traditional classifiers.

Question 7. Our traditional and hierarchical classification approaches both struggled with recognizing underrepresented postures. The final question this thesis addresses is whether a multi-class boosting method, specifically a confusion matrix boosting algorithm, successfully combats the class-imbalance problem within our dataset. Our experiments show great promise with confusion matrix boosting as it proves to be successful when trained by activity domain.

Overall, we developed a dataset containing almost 100 hours of direct observation from three novel sets of accelerometer data collected by the Cal Poly Kinesiology and Public Health Department. This dataset is structured in a way that lays out multiple directions for future work. We were successful in detecting the amount of time an individual spends performing an activity. Across the board on all coding schemes, we found the BioStamp monitors were much better indicators of physical activity on a second-by-second basis than the wrist-worn Actigraph. This is most likely due to the amount of noise the wrist accelerometer experiences while it is worn. One exception to this conclusion would be in our sedentary versus non-sedentary experiments, where the chest BioStamp performed similarly to the wrist. Out of all of the machine learn- 
ing techniques we tested on our accelerometer data, random forests and $k$-nearest neighbors both performed comparably and were the best estimators of behavior for each activity monitor. Furthermore, our hierarchical classification schema was able to reduce the severity of error from our traditional random forest classifiers on some monitors. Finally, we made an effort to better predict classes that are underrepresented in our dataset using the confusion matrix boosting algorithm proposed by [34], which achieved promising preliminary results in our experiments. 


\section{Chapter 7}

\section{FUTURE WORK}

This work trains models on a novel free-living acclerometer dataset and compares performance to that of a laboratory-trained model. There are many other publiclyavailable activity recognition methods that would be interesting to include in a sideby-side performance comparison. Future work should test previously developed models on our data so that the performance of our models can be evaluated alongside others. Our work also estimates physical activity on a second-by-second level. Prior work has used larger time intervals, ranging from 15 seconds to one minute. In order to more directly compare performance with other methods, future work should evaluate our models using larger time windows.

We used sci-kit learn's AdaBoost and gradient boosting classifiers as an attempt to improve classification accuracy. Given the imbalanced nature of our dataset, we also implemented the CoMBo algorithm [34] in an effort to combat the class-imbalance problem. Future work should evaluate our models on a more balanced dataset. Another direction for future work would be to investigate developing a boosting method that uses domain knowledge about the activity classes to reweigh the dataset after each classification attempt rather than the standard reweighing technique AdaBoost uses. This boosting method could reweigh the misclassified data points by using prior domain knowledge about the importance of detecting certain misclassification situations. For example, according to Dr. Keadle, misclassifying sitting as running is absolutely unacceptable, but the misclassification of sitting for standing is less of an issue, because the activities are more closely related. In this scenario, the weights for the sitting data points that were misclassified as running would be greater than the weights of the sitting points that were misclassified as standing due to a hard-coded 
rule that sitting being misclassified as running is a more important problem than sitting being misclassified as standing.

In addition, when creating our ground truth dataset, we added a column that labels seconds that contain more than one posture, which we refer to as transition seconds. For most of our experiments, we excluded these seconds in order to provide our models with "purer" training data. Future work should investigate training models to learn seconds in which multiple postures are observed.

Finally, future work should build models to learn riding in a vehicle. This may potentially require studies to try to "control" the nature of free-living data by collecting more samples of riding in a vehicle. 


\section{BIBLIOGRAPHY}

[1] American Diabetes Association. Statistics About Diabetes. http://www.diabetes.org/diabetes-basics/statistics/. Date accessed: January 2018.

[2] American Heart Association. Heart Disease and Stroke Statistics - At-a-Glance, 2015. http://www .heart.org/idc/groups/ahamahpublic/@wcm/@sop/@smd/documents/downloadable/ucm_470704.pdf.

[3] K. Aminian, P. Robert, E. Buchser, B. Rutschmann, D. Hayoz, and M. Depairon. Physical activity monitoring based on accelerometry: Validation and comparison with video observation. Med. Biol. Eng. Comput., 37:304-308, 1999.

[4] A. Bayat, M. Pomplun, and D. A. Tran. A study on human activity recognition using accelerometer data from smartphones. Procedia Computer Science, 34(C):450-457, 2014.

[5] S. N. Blair, Y. Cheng, and J. S. Holder. Is physical activity or physical fitness more important in defining health benefits? Med Sci Sports Exerc., 2000.

[6] A. G. Bonomi, A. H. Goris, B. Yin, and K. R. Westerterp. Detection of Type, Duration, and Intensity of Physical Activity Using an Accelerometer. Medicine E Science in Sports \& Exercise, 41(9):1770-1777, 2009.

[7] L. Breiman. Random forests. Machine Learning, 45(1):5-32, Oct 2001.

[8] A. G. Brooks, R. T. Withers, C. J. Gore, A. G. Vogler, J. Plummer, and J. Cormack. Measurement and prediction of mets during household activities in 35- to 45-year-old females. Eur J Appl Physiol, 91:638-648, 2004. 
[9] Bureau of Labor Statistics. American Time Use Survey Activity Lexicon, 2016. https://www.bls.gov/tus/lexiconwex2016.pdf.

[10] Center for Disease Control and Prevention. Overweight $\mathscr{G}$ Obesity, Aug 2018. https://www.cdc.gov/obesity/data/adult.html.

[11] A. K. Chowdhury, D. Tjondronegoro, V. Chandran, and S. G. Trost. Ensemble Methods for Classification of Physical Activities from Wrist Accelerometry. Medicine and Science in Sports and Exercise, 49(9):1965-1973, 2017.

[12] C. Cortes and V. Vapnik. Support-vector networks. Machine Learning, 20(3):273-297, Sep 1995.

[13] S. E. Crouter, E. Kuffel, J. D. Haas, E. A. Frongillo, and D. R. Bassett Jr. Refined two-regression model for the actigraph accelerometer. Med Sci Sports Exerc, 42:1029-1037, 2010.

[14] T. Daghistani and R. Alshammari. Improving Accelerometer-Based Activity Recognition by Using Ensemble of Classifiers. IJACSA) International Journal of Advanced Computer Science and Applications, 7(5), 2016.

[15] S. De Vries, F. G. Garre, L. H. Engbers, V. H. Hildebrandt, and S. Van Buuren. Evaluation of neural networks to identify types of activity using accelerometers. Medicine and Science in Sports and Exercise, 43(1):101-107, 2011.

[16] S. Eglowski. Create: Clinical record analysis technology ensemble. Master's thesis, California Polytechnic State University, San Luis Obispo, June 2017.

[17] K. Ellis, S. Godbole, J. Chen, S. Marshall, G. Lanckriet, and J. Kerr. Physical activity recognition in free-living from body-worn sensors. In Proceedings of the 4th International SenseCam; Pervasive Imaging Conference, SenseCam '13, pages 88-89, New York, NY, USA, 2013. ACM. 
[18] K. Ellis, S. Godbole, S. Marshall, G. R. G. Lanckriet, J. Staudenmayer, and J. Kerr. Identifying active travel behaviors in challenging environments using gps, accelerometers, and machine learning algorithms. In Front. Public Health, 2014.

[19] K. Ellis, J. Kerr, S. Godbole, G. Lanckriet, D. Wing, and S. Marshall. A random forest classifier for the prediction of energy expenditure and type of physical activity from wrist and hip accelerometers. 51(1):87-100, 2014.

[20] K. Ellis, J. Kerr, S. Godbole, J. Staudenmayer, and G. Lanckriet. Hip and Wrist Accelerometer Algorithms for Free-Living Behavior Classification. Med Sci Sports Exerc., 344(6188):1173-1178, 2015.

[21] M. Ermes, J. Pärkkä, J. Mäntyjärvi, and I. Korhonen. Detection of daily activities and sports with wearable sensors in controlled and uncontrolled conditions. IEEE Transactions on Information Technology in Biomedicine, 2008.

[22] F. Foerster and J. Fahrenberg. Motion pattern and posture: Correctly assessed by calibrated accelerometers. Behav. Res. Methods Instrum. Comput., $32(3): 450-457,2000$.

[23] F. Foerster, M. Smeja, and J. Fahrenberg. Detection of posture and motion by accelerometry: A validation study in ambulatory monitoring. Comput. Human Behav., 15:571-583, 1999.

[24] P. S. Freedson, E. Melanson, and J. Sirard. Calibration of the computer science and applications, inc. accelerometer. Med Sci Sports Exerc, 30:777-781, 1998.

[25] Y. Freund and R. E. Schapire. Experiments with a new boosting algorithm. In Proceedings of the Thirteenth International Conference on International 
Conference on Machine Learning, ICML'96, pages 148-156, San Francisco, CA, USA, 1996. Morgan Kaufmann Publishers Inc.

[26] I. Goodfellow, Y. Bengio, and A. Courville. Deep Learning. MIT Press, 2016. http://www. deeplearningbook.org.

[27] I. C. Gyllensten and A. G. Bonomi. Identifying types of physical activity with a single accelerometer: Evaluating laboratory-trained algorithms in daily life. IEEE Transactions on Biomedical Engineering, 58(9):2656-2663, 2011.

[28] W. L. Haskell, I.-M. Lee, R. R. Pate, K. E. Powell, and S. N. Blair. Physical Activity and Public Health: Updated Recommendation for Adults From the American College of Sports Medicine and the American Heart Association. Circulation CirculationMedicine and Science in Sports and Exercise, 116(398):1081-1093, 2007.

[29] D. Karantonis, M. Narayanan, M. Mathie, N. Lovell, and B. Celler. Implementation of a real-time human movement classifier using a triaxial accelerometer for ambulatory monitoring. IEEE Trans. Inf. Technol. Biomed., 10:156-157, Jan 2006.

[30] R. J. Kate, A. M. Swartz, W. A. Welch, and S. J. Strath. Comparative evaluation of features and techniques for identifying activity type and estimating energy cost from accelerometer data. Physiological Measurement, 37(3):360-379, 2016.

[31] J. Kerr, S. Marshall, S. Godbole, J. Chen, A. Legge, A. R Doherty, P. Kelly, M. Smith, H. Badland, and C. Foster. Using the sensecam to improve classifications of sedentary behavior in free-living settings. 44:290-6, 032013.

[32] P. KL, T. RP, B. RM, and et al. The physical activity guidelines for americans. JAMA, 320(19):2020-2028, 2018. 
[33] S. Kozey-Keadle, A. Libertine, K. Lyden, J. Staudenmayer, and P. S. Freedson. Validation of Wearable Monitors for Assessing Sedentary Behavior. Med. Sci. Sports Exerc, 43(8):1561-1567, 2011.

[34] S. Koo and C. Capponi. On multi-class classification through the minimization of the confusion matrix norm. In $A C M L, 2013$.

[35] S. Kullback and R. A. Leibler. On information and sufficiency. Ann. Math. Statist., 22(1):79-86, 031951.

[36] C. Li. Wearable Computing: Accelerometer-Based Human Activity Classification Using Decision Tree. Master's thesis, Utah State University, 2017.

[37] K. Lyden, S. K. Keadle, J. Staudenmayer, and P. S. Freedson. A Method to Estimate Free-Living Active and Sedentary Behavior from an Accelerometer. Medicine $\mathcal{E}$ Science in Sports 83 Exercise, 46(2):386-397, Feb 2014.

[38] A. Mannini, S. S. Intille, M. Rosenberger, A. M. Sabatini, and W. Haskell. Activity recognition using a single accelerometer placed at the wrist or ankle. 45(11):2193-2203, 2014.

[39] M. Mathie, A. Foster, N. Lovell, and B. Celler. Detection of daily physical activities using a triaxial accelerometer. Med. Biol. Eng. Comput., 37:296-301, 2003.

[40] U. Maurer, A. Smailagic, D. Siewiorek, and M. Deisher. Activity recognition and monitoring using multiple sensors on different body positions. presented at the Int. Workshop Wearable Implantable Body Sensor Netw. Cambridge, MA, 2006.

[41] Y. Mehra, G. Moir, A. Rose, and H. Schumann. Data 451 final report. 2017. 
[42] I. Mukherjee and R. E. Schapire. A theory of multiclass boosting. J. Mach. Learn. Res., 14(1):437-497, Feb. 2013.

[43] J. Ng, A. Sahakian, and S. Swiryn. Accelerometer-based body-position sensing for ambulatory electrocardiographic monitoring. Biomed. Instrum. Technol., 37:338-346, 2003.

[44] OpenCV. Introduction to support vector machines.

[45] N. Owen, G. N. Healy, C. E. Matthews, and D. W. Dunstan. Too much sitting: The population health science of sedentary behavior. Exercise and Sport Sciences Reviews, 38(3):105-113, jul 2010.

[46] B. C. Paes, A. Plastino, and A. A. Freitas. Improving Local Per Level Hierarchical Classification. Journal of Information and Data Management, (3):394-409, October 2012. http://citeseerx.ist.psu.edu/viewdoc/ download?doi=10.1.1.308.8321\{\&\}rep=rep $1\{\&\}$ type=pdf.

[47] S. Patel. Chapter 4: K nearest neighbors classifier - machine learning 101 medium, May 2017.

[48] S. Patel, R. S. McGinnis, I. Silva, S. DiCristofaro, N. Mahadevan, E. Jortberg, J. Franco, A. Martin, J. Lust, M. Raj, B. McGrane, P. DePetrillo, A. J. Aranyosi, M. Ceruolo, J. Pindado, and R. Ghaffari. A wearable computing platform for developing cloud-based machine learning models for health monitoring applications. 2016 38th Annual International Conference of the IEEE Engineering in Medicine and Biology Society (EMBC), (1):5997-6001, 2016.

[49] F. Pedregosa, G. Varoquaux, A. Gramfort, V. Michel, B. Thirion, O. Grisel, M. Blondel, P. Prettenhofer, R. Weiss, V. Dubourg, J. Vanderplas, A. Passos, 
D. Cournapeau, M. Brucher, M. Perrot, and E. Duchesnay. Scikit-learn: Machine learning in Python. Journal of Machine Learning Research, $12: 2825-2830,2011$.

[50] G. Plasqui and K. R. Westerterp. Physical Activity Assessment With Accelerometers: An Evaluation Against Doubly Labeled Water.

[51] S. J. Preece, J. Y. Goulermas, L. P. J. Kenney, D. Howard, K. Meijer, and R. Crompton. Activity identification using body-mounted sensorsa review of classification techniques. Physiological Measurement, 30(4):R1-R33, 2009.

[52] J. Quinlan. C4.5: Program for Machine Learning. Morgan Kaufman, 1992.

[53] Shaopeng Liu, R. X. Gao, D. John, J. Staudenmayer, and P. S. Freedson. SVM-based multi-sensor fusion for free-living physical activity assessment. In 2011 Annual International Conference of the IEEE Engineering in Medicine and Biology Society, pages 3188-3191. IEEE, aug 2011.

[54] C. Silla and A. Freitas. A survey of hierarchical classification across different application domains. Data Mining and Knowledge Discovery, 22:31-72, January 2011.

[55] J. Skotte, M. Korshøj, J. Kristiansen, C. Hanisch, and A. Holtermann. Detection of Physical Activity Types Using Triaxial Accelerometers. Journal of Physical Activity and Health, 11:76-84, 2014.

[56] J. Staudenmayer, S. He, A. Hickey, J. Sasaki, and P. Freedson. Methods to estimate aspects of physical activity and sedentary behavior from high-frequency wrist accelerometer measurements., Aug 2015. https://www.ncbi.nlm.nih.gov/pubmed/26112238. 
[57] J. Staudenmayer, D. Pober, S. Crouter, D. Bassett, and P. Freedson. An artificial neural network to estimate physical activity energy expenditure and identify physical activity type from an accelerometer. J Appl Physiol, pages 1300-1307, 2009.

[58] M. Sufyian, M. Azmi, and M. N. Sulaiman. Accelerator-Based Human Activity Recognition Using Voting Technique with NBTree and MLP Classifiers. 7(1), 2017.

[59] M. M. Takeda, J. Martinez, and S. K. Keadle. Comparing hip and wrist accelerometer estimates of moderate-vigorous physical activity across activity domains. 2017.

[60] S. Trost, P. Loprinzi, R. Moore, and K. Pfeiffer. Comparison of accelerometer cut points for predicting activity intensity in youth. 43:1360-8, 122010.

[61] K. Zhang, P. Werner, M. Sun, F. X. Pi-Sunyer, and C. N. Boozer. Measurement of Human Daily Physical Activity. Obesity Research, 11(1):33-40, jan 2003.

[62] J. Zhu, S. Rosset, H. Zou, and T. Hastie. Multi-class AdaBoost, January 2006. 


\section{APPENDICES}

Appendix A

\section{DATASET SAMPLES}

\begin{tabular}{|c|c|c|c|c|c|c|c|c|}
\hline ID & session & type & start_month & start_day & start_year & Actual Start & Actual Stop & Duration \\
\hline AM01 & DO1 & L & 10 & 3 & 2017 & $18: 44: 45$ & $20: 47: 00$ & 2:02:15 \\
\hline AM01 & DO2 & $\mathrm{H}$ & 10 & 6 & 2017 & $16: 43: 57$ & $18: 45: 00$ & 2:01:03 \\
\hline AM02 & DO1 & $\mathrm{H}$ & 7 & 24 & 2017 & $13: 17: 10$ & $15: 17: 32$ & $2: 00: 22$ \\
\hline AM02 & DO2_a & A & 7 & 25 & 2017 & $8: 00: 27$ & $8: 52: 32$ & $0: 52: 05$ \\
\hline AM02 & DO2_b & A & 7 & 25 & 2017 & $8: 56: 13$ & $10: 12: 29$ & $1: 16: 16$ \\
\hline AM03 & DO1 & L & 7 & 25 & 2017 & $14: 00: 10$ & $16: 00: 23$ & $2: 00: 13$ \\
\hline AM03 & DO2 & $\mathrm{H}$ & 7 & 27 & 2017 & $13: 01: 29$ & $15: 00: 39$ & 1:59:10 \\
\hline AM04 & DO1 & W & 9 & 12 & 2017 & $12: 02: 56$ & $14: 04: 00$ & 2:01:04 \\
\hline AM04 & $\mathrm{DO} 2$ & A & 9 & 17 & 2017 & $14: 15: 58$ & $16: 16: 00$ & 2:00:02 \\
\hline AM05 & DO2 & L & 9 & 19 & 2017 & $19: 38: 53$ & $21: 40: 00$ & 2:01:07 \\
\hline AM06 & DO1 & $E$ & 7 & 31 & 2017 & $8: 35: 23$ & $10: 36: 03$ & $2: 00: 40$ \\
\hline AM06 & DO2 & A & 8 & 1 & 2017 & $8: 09: 22$ & $10: 10: 03$ & 2:00:41 \\
\hline AM07 & DO1 & $\mathrm{H}$ & 8 & 2 & 2017 & 9:01:18 & $11: 01: 27$ & 2:00:09 \\
\hline AM07 & DO2 & $\mathrm{E}$ & 8 & 2 & 2017 & $11: 30: 08$ & $13: 30: 19$ & 2:00:11 \\
\hline AM08 & DO1_a & $\mathrm{E}$ & 8 & 3 & 2017 & 9:08:47 & $10: 24: 09$ & $1: 15: 22$ \\
\hline AM08 & D01_b & $E$ & 8 & 3 & 2017 & $10: 26: 00$ & $11: 10: 52$ & $0: 44: 52$ \\
\hline AM08 & DO2 & A & 8 & 7 & 2017 & $9: 30: 17$ & $11: 30: 47$ & $2: 00: 30$ \\
\hline AM09 & DO1 & W & 8 & 10 & 2017 & $13: 26: 00$ & $15: 08: 44$ & $1: 42: 44$ \\
\hline AM09 & DO2 & A & 8 & 10 & 2017 & $16: 18: 04$ & $18: 18: 36$ & $2: 00: 32$ \\
\hline
\end{tabular}

Figure A.1: A snapshot of the direct observation timestamp log as provided by the Cal Poly Kinesiology and Public Health Department. 


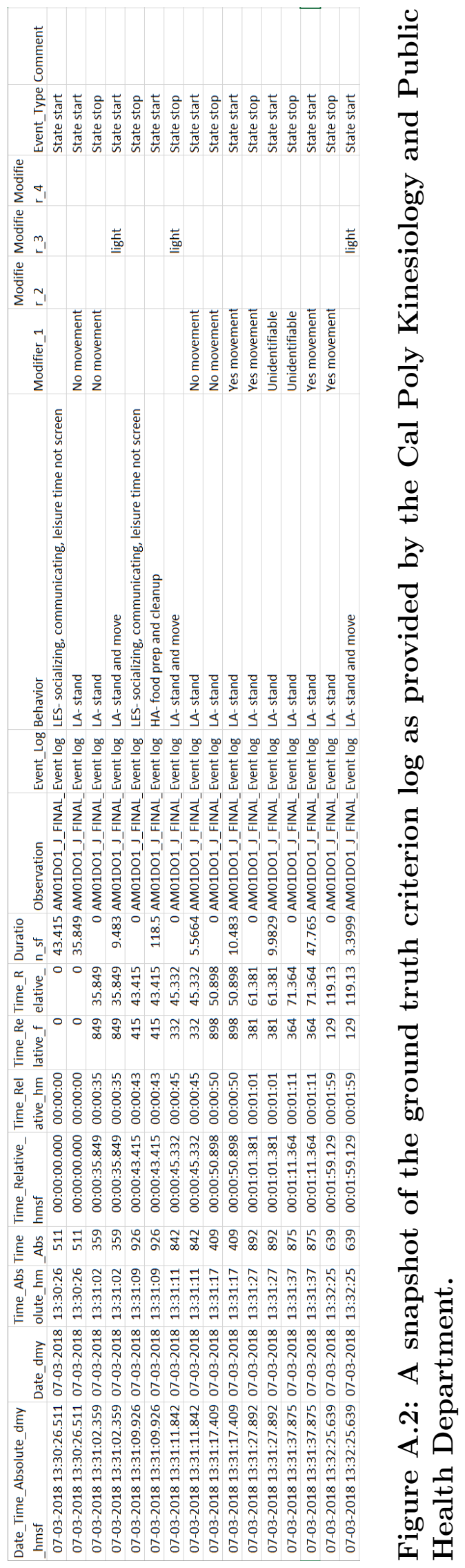




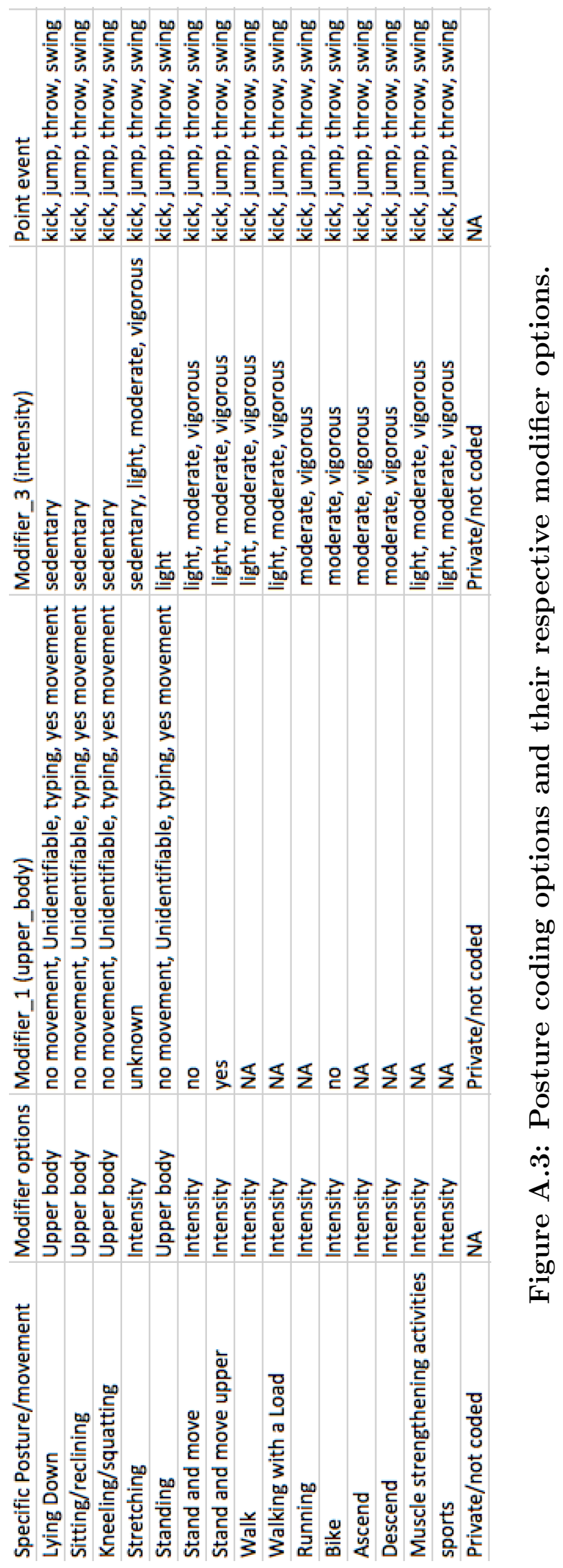


Appendix B

CONFUSION MATRICES

\begin{tabular}{|c|c|c|c|c|c|}
\hline & \multicolumn{5}{|c|}{ Predicted } \\
\hline \multirow{4}{*}{ Actual } & & sedentary & light & moderate & vigorous \\
\cline { 2 - 6 } & sedentary & $\mathbf{1 1 2 3 1 3}$ & 48393 & 10172 & 958 \\
\cline { 2 - 6 } & light & 49610 & $\mathbf{4 4 4 0 5}$ & 12609 & 1316 \\
\cline { 2 - 6 } & moderate & 13365 & 15739 & $\mathbf{1 5 0 1 9}$ & 2325 \\
\cline { 2 - 6 } & vigorous & 1693 & 3316 & 3339 & $\mathbf{6 8 7 6}$ \\
\hline
\end{tabular}

Table B.1: Confusion matrix for retrained random forest model predicting METs from wrist Actigraph.

\begin{tabular}{|c|c|c|c|c|c|}
\hline & \multicolumn{5}{|c|}{ Predicted } \\
\hline \multirow{4}{*}{ Actual } & & sedentary & light & moderate & vigorous \\
\cline { 2 - 6 } & sedentary & $\mathbf{1 6 9 0 2 1}$ & 2626 & 342 & 21 \\
\cline { 2 - 6 } & light & 1406 & $\mathbf{1 0 6 4 8 2}$ & 4171 & 219 \\
\cline { 2 - 6 } & moderate & 361 & 7174 & $\mathbf{3 8 8 1 4}$ & 187 \\
\cline { 2 - 6 } & vigorous & 410 & 1345 & 610 & $\mathbf{1 2 8 7 1}$ \\
\hline
\end{tabular}

Table B.2: Confusion matrix for retrained random forest model predicting METs from thigh BioStamp.

\begin{tabular}{|c|c|c|c|c|c|}
\hline & \multicolumn{5}{|c|}{ Predicted } \\
\hline \multirow{4}{*}{ Actual } & & sedentary & light & moderate & vigorous \\
\cline { 2 - 6 } & sedentary & $\mathbf{1 6 9 0 4 0}$ & 2621 & 319 & 30 \\
\cline { 2 - 6 } & light & 1373 & $\mathbf{1 0 6 5 8 7}$ & 4082 & 236 \\
\cline { 2 - 6 } & moderate & 342 & 7291 & $\mathbf{3 8 7 2 4}$ & 179 \\
\cline { 2 - 6 } & vigorous & 876 & 2492 & 310 & $\mathbf{1 1 5 5 8}$ \\
\hline
\end{tabular}

Table B.3: Confusion matrix for retrained random forest predicting METs from chest BioStamp. 


\begin{tabular}{|c|c|c|c|c|c|}
\hline & \multicolumn{5}{|c|}{ Predicted } \\
\hline \multirow{4}{*}{ Actual } & & sedentary & light & moderate & vigorous \\
\cline { 2 - 6 } & sedentary & $\mathbf{1 6 8 8 8 7}$ & 2752 & 332 & 39 \\
\cline { 2 - 6 } & light & 1441 & $\mathbf{1 0 6 6 0 5}$ & 3997 & 235 \\
\cline { 2 - 6 } & moderate & 353 & 7137 & $\mathbf{3 8 8 7 6}$ & 170 \\
\cline { 2 - 6 } & vigorous & 348 & 2059 & 266 & $\mathbf{1 2 5 6 3}$ \\
\hline
\end{tabular}

Table B.4: Confusion matrix for retrained random forest predicting METs from thigh and chest BioStamps.

\begin{tabular}{|c|c|c|c|c|}
\hline & \multicolumn{4}{|c|}{ Predicted } \\
\hline \multirow{4}{*}{ Actual } & & Sedentary & Non-sedentary & Recall \\
\cline { 2 - 5 } & Sedentary & $\mathbf{4 6 9 7 7}$ & 3342 & 0.934 \\
\cline { 2 - 5 } & Non-sedentary & 5867 & $\mathbf{1 7 8 9 5}$ & 0.753 \\
\cline { 2 - 5 } & Precision & 0.889 & 0.843 & 0.876 \\
\hline
\end{tabular}

Table B.5: Confusion matrix for RF predicting sedentary time from wrist Actigraph on active observation sessions.

\begin{tabular}{|c|c|c|c|c|}
\hline & \multicolumn{4}{|c|}{ Predicted } \\
\hline \multirow{4}{*}{ Actual } & & Sedentary & Non-sedentary & Recall \\
\cline { 2 - 5 } & Sedentary & $\mathbf{3 3 7 1 6}$ & 17231 & 0.662 \\
\cline { 2 - 5 } & Non-sedentary & 7538 & $\mathbf{9 9 5 8}$ & 0.569 \\
\cline { 2 - 5 } & Precision & 0.817 & 0.366 & 0.638 \\
\hline
\end{tabular}

Table B.6: Confusion matrix for RF predicting sedentary time from wrist Actigraph on errands observation sessions.

\begin{tabular}{|c|c|c|c|c|}
\hline & \multicolumn{4}{|c|}{ Predicted } \\
\hline \multirow{4}{*}{ Actual } & & Sedentary & Non-sedentary & Recall \\
\cline { 2 - 5 } & Sedentary & $\mathbf{1 1 1 1 8}$ & 8210 & 0.575 \\
\cline { 2 - 5 } & Non-sedentary & 11999 & $\mathbf{3 7 9 3 7}$ & 0.76 \\
\cline { 2 - 5 } & Precision & 0.481 & 0.822 & 0.708 \\
\hline
\end{tabular}

Table B.7: Confusion matrix for RF predicting sedentary time from wrist Actigraph on work observation sessions.

\begin{tabular}{|c|c|c|c|c|}
\hline & \multicolumn{4}{|c|}{ Predicted } \\
\hline \multirow{4}{*}{ Actual } & & Sedentary & Non-sedentary & Recall \\
\cline { 2 - 5 } & Sedentary & $\mathbf{4 6 1 7}$ & 954 & 0.829 \\
\cline { 2 - 5 } & Non-sedentary & 10125 & $\mathbf{5 2 0 7 9}$ & 0.837 \\
\cline { 2 - 5 } & Precision & 0.313 & 0.982 & 0.837 \\
\hline
\end{tabular}

Table B.8: Confusion matrix for RF predicting sedentary time from wrist Actigraph on leisure observation sessions. 


\begin{tabular}{|c|c|c|c|c|}
\hline & \multicolumn{4}{|c|}{ Predicted } \\
\hline \multirow{4}{*}{ Actual } & & Sedentary & Non-sedentary & Recall \\
\cline { 2 - 5 } & Sedentary & $\mathbf{3 9 0 6 3}$ & 11120 & 0.778 \\
\cline { 2 - 5 } & Non-sedentary & 6369 & $\mathbf{1 4 8 7 3}$ & 0.7 \\
\cline { 2 - 5 } & Precision & 0.86 & 0.572 & 0.755 \\
\hline
\end{tabular}

Table B.9: Confusion matrix for RF predicting sedentary time from wrist Actigraph on household observation sessions.

\begin{tabular}{|c|c|c|c|c|}
\hline & \multicolumn{4}{|c|}{ Predicted } \\
\hline \multirow{4}{*}{ Actual } & & Sedentary & Non-sedentary & Recall \\
\cline { 2 - 5 } & Sedentary & $\mathbf{5 0 0 3 5}$ & 284 & 0.994 \\
\cline { 2 - 5 } & Non-sedentary & 575 & $\mathbf{2 3 1 8 7}$ & 0.976 \\
\cline { 2 - 5 } & Precision & 0.989 & 0.988 & 0.989 \\
\hline
\end{tabular}

Table B.10: Confusion matrix for RF predicting sedentary time from thigh and chest BioStamps on active observation sessions.

\begin{tabular}{|c|c|c|c|c|}
\hline & \multicolumn{4}{|c|}{ Predicted } \\
\hline \multirow{4}{*}{ Actual } & & Sedentary & Non-sedentary & Recall \\
\cline { 2 - 5 } & Sedentary & $\mathbf{5 5 0 4 8}$ & 287 & 0.995 \\
\cline { 2 - 5 } & Non-sedentary & 581 & $\mathbf{1 6 9 1 5}$ & 0.967 \\
\cline { 2 - 5 } & Precision & 0.99 & 0.983 & 0.988 \\
\hline
\end{tabular}

Table B.11: Confusion matrix for RF predicting sedentary time from thigh and chest BioStamps on errands observation sessions.

\begin{tabular}{|c|c|c|c|c|}
\hline & \multicolumn{4}{|c|}{ Predicted } \\
\hline \multirow{4}{*}{ Actual } & & Sedentary & Non-sedentary & Recall \\
\cline { 2 - 5 } & Sedentary & $\mathbf{1 8 9 4 2}$ & 386 & 0.98 \\
\cline { 2 - 5 } & Non-sedentary & 548 & $\mathbf{4 9 0 5 3}$ & 0.989 \\
\cline { 2 - 5 } & Precision & 0.972 & 0.992 & 0.986 \\
\hline
\end{tabular}

Table B.12: Confusion matrix for RF predicting sedentary time from thigh and chest BioStamps on work observation sessions.

\begin{tabular}{|c|c|c|c|c|}
\hline & \multicolumn{4}{|c|}{ Predicted } \\
\hline \multirow{4}{*}{ Actual } & & Sedentary & Non-sedentary & Recall \\
\cline { 2 - 5 } & Sedentary & $\mathbf{5 4 2 7}$ & 144 & 0.974 \\
\cline { 2 - 5 } & Non-sedentary & 189 & $\mathbf{6 2 0 1 5}$ & 0.997 \\
\cline { 2 - 5 } & Precision & 0.966 & 0.998 & 0.995 \\
\hline
\end{tabular}

Table B.13: Confusion matrix for RF predicting sedentary time from thigh and chest BioStamps on leisure observation sessions. 


\begin{tabular}{|c|c|c|c|c|}
\hline & \multicolumn{4}{|c|}{ Predicted } \\
\hline \multirow{4}{*}{ Actual } & & Sedentary & Non-sedentary & Recall \\
\cline { 2 - 5 } & Sedentary & $\mathbf{4 9 2 0 2}$ & 981 & 0.98 \\
\cline { 2 - 5 } & Non-sedentary & 1205 & $\mathbf{1 9 9 0 8}$ & 0.943 \\
\cline { 2 - 5 } & Precision & 0.976 & 0.953 & 0.969 \\
\hline
\end{tabular}

Table B.14: Confusion matrix for RF predicting sedentary time from thigh and chest BioStamps on household observation sessions. 


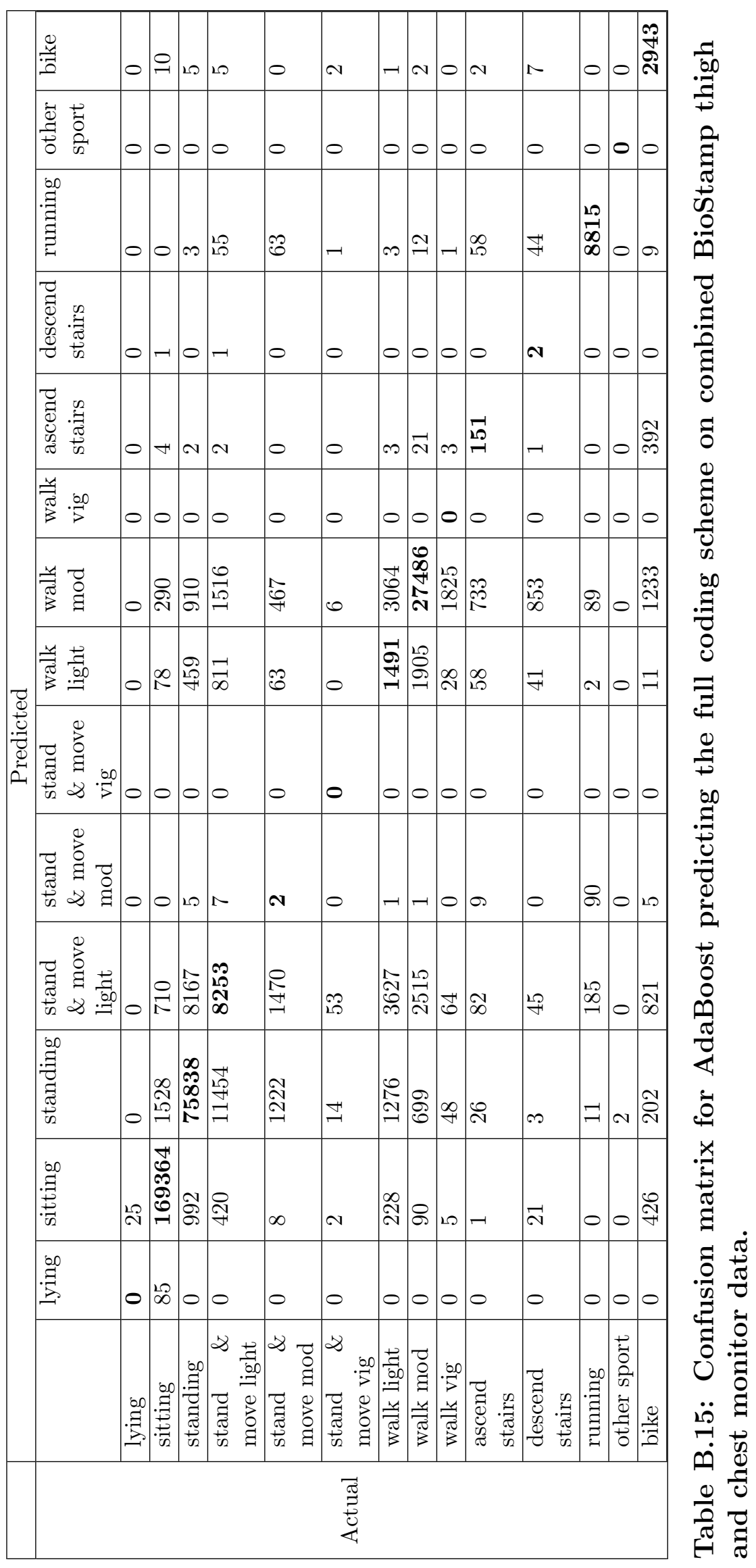


Appendix C

MODEL ACCURACIES

\begin{tabular}{|l|l|l|l|l|}
\hline \multirow{2}{*}{ Model } & \multicolumn{3}{|c|}{ Accuracy (\%) } \\
\cline { 2 - 5 } & $\begin{array}{l}\text { Actigraph } \\
\text { Wrist }\end{array}$ & $\begin{array}{l}\text { BioStamp Thigh } \\
\text { and Chest }\end{array}$ & $\begin{array}{l}\text { BioStamp } \\
\text { Thigh }\end{array}$ & $\begin{array}{l}\text { BioStamp } \\
\text { Chest }\end{array}$ \\
\hline \hline $\begin{array}{l}\text { Random Forest with } \\
\text { transitions }\end{array}$ & 61.41 & 84.13 & 84.03 & 84.12 \\
\hline $\begin{array}{l}\text { Random Forest with- } \\
\text { out transitions } \\
\text { Hierarchy }\end{array}$ & 61.61 & 85.98 & 85.73 & 85.47 \\
\hline
\end{tabular}

Table C.1: Test accuracy comparison of random forests with transition seconds in the dataset vs. excluding transition seconds from the dataset on the full coding scheme across all monitors.

\begin{tabular}{|l|l|l|l|l|}
\hline \multirow{2}{*}{ Model } & \multicolumn{3}{|c|}{ Accuracy (\%) } \\
\cline { 2 - 5 } & $\begin{array}{l}\text { Actigraph } \\
\text { Wrist }\end{array}$ & $\begin{array}{l}\text { BioStamp Thigh } \\
\text { and Chest }\end{array}$ & $\begin{array}{l}\text { BioStamp } \\
\text { Thigh }\end{array}$ & $\begin{array}{l}\text { BioStamp } \\
\text { Chest }\end{array}$ \\
\hline $\begin{array}{l}\text { Random Forest } \\
\text { maxdepth }=5\end{array}$ & 76.42 & 95.54 & 98.51 & 77.17 \\
\hline $\begin{array}{l}\text { Random Forest } \\
\text { maxdepth }=15\end{array}$ & 76.12 & 98.5 & 98.45 & 76.34 \\
\hline
\end{tabular}

Table C.2: Test accuracy comparison of random forests using different maximum tree depths on the sedentary coding scheme.

\begin{tabular}{|l|l|l|l|l|}
\hline \multirow{2}{*}{ Model } & \multicolumn{4}{|c|}{ Accuracy (\%) } \\
\cline { 2 - 5 } & $\begin{array}{l}\text { Actigraph } \\
\text { Wrist KNN }\end{array}$ & $\begin{array}{l}\text { Actigraph } \\
\text { Wrist RF }\end{array}$ & $\begin{array}{l}\text { BioStamp Thigh } \\
\text { and Chest SVM }\end{array}$ & $\begin{array}{l}\text { BioStamp Thigh } \\
\text { and Chest RF }\end{array}$ \\
\hline \hline 7 Features & 62.55 & 76.42 & 89.44 & 98.54 \\
\hline 23 Features & 63.72 & 77.17 & 95.77 & 98.6 \\
\hline
\end{tabular}

Table C.3: Test accuracy comparison of models with different number of total features on the sedentary coding scheme. 